PROGRAMS AND ACTIVITIES OF THE

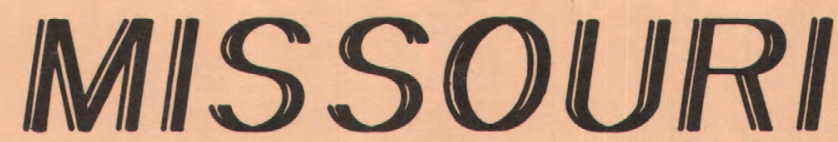

DISTRICT, WATER RESOURCES DIVISION UNITED STATES GEOLOGICAL SURVEY
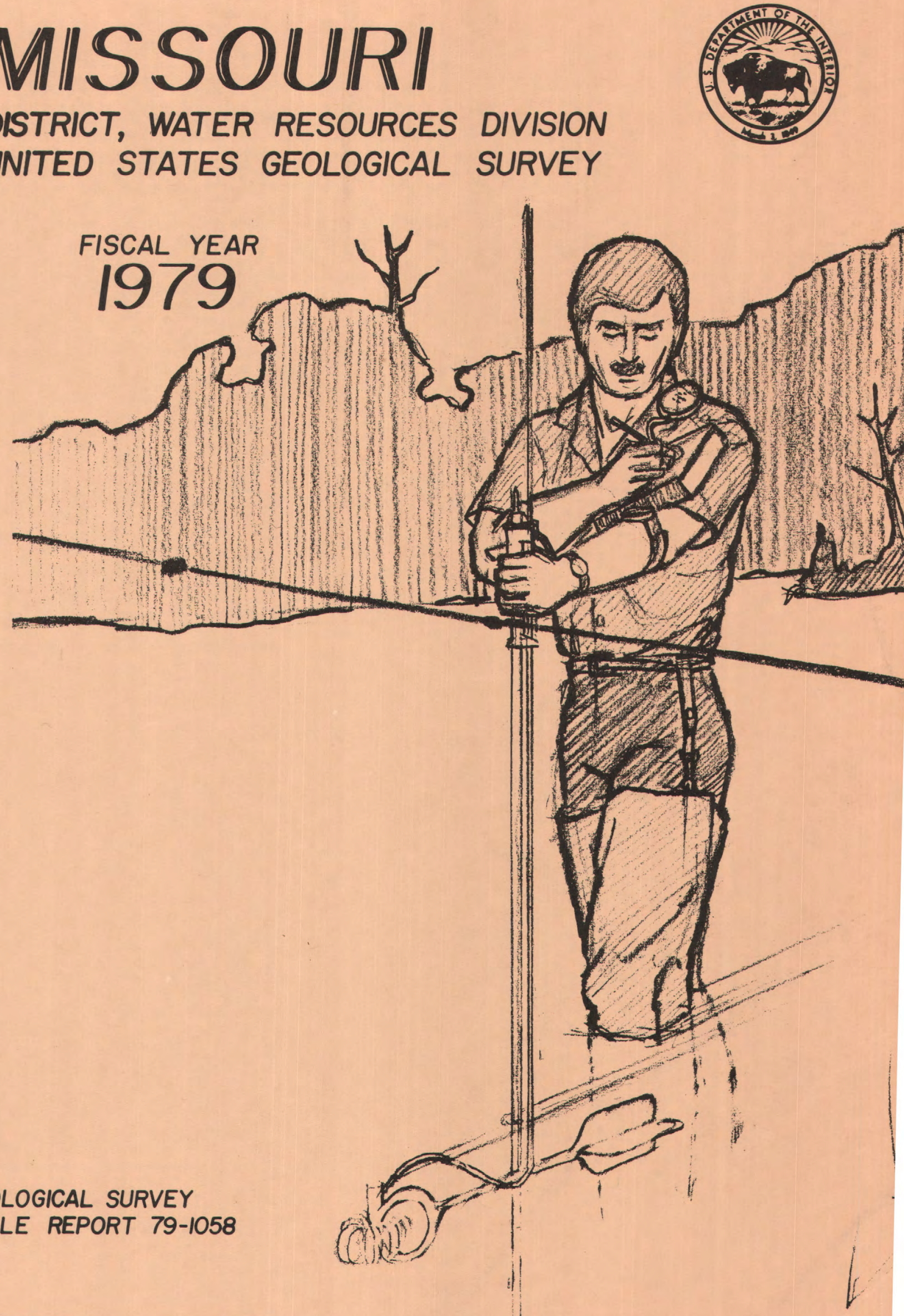

U.S. GEOLOGICAL SURVEY

OPEN-FILE REPORT 79-1058 
Front cover drawing by

Douglas R. Stark,

Division of Geology and Land Survey 


\section{PROGRAMS AND ACTIVITIES OF THE \\ MISSOURI DISTRICT, WATER RESOURCES DIVISION, \\ U.S. GEOLOGICAL SURVEY}

\section{FISCAL YEAR 1979}

Compiled by Wanietia M. Kratzer

U.S. GEOLOGICAL SURVEY

Open-File Report 79-1058

Rolla, Missouri

July 1979 
UNITED STATES DEPARTMENT OF THE INTERIOR

CECIL D. ANDRUS, Secretary

GEOLOGICAL SURVEY

H. William Menard, Director

Water Resources Division

0. Milton Hackett, Acting Chief Hydrologist

For additional information write to:

Don L. Coffin, District Chief

U.S. Geological Survey, Water Resources Division

1400 Independence Road Mail Stop 200

Rolla, Missouri 65401 
Abstract---1-1-1

Introduction--1-1

Cooperation--1-1-1

Hydrologic-data program--a-- 4

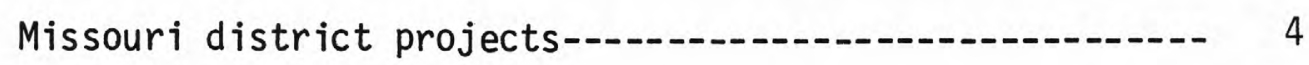

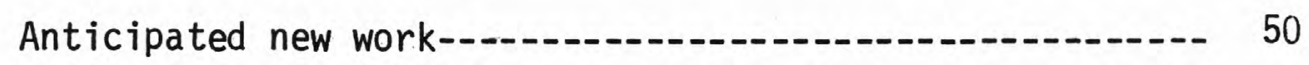

Sources of information--1-1-1 51

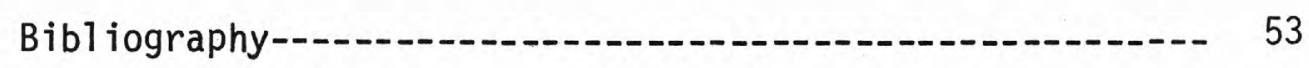




\section{ILLUSTRATIONS}

\section{Page}

Figure 1. Chart showing organization of the Missouri district--.-_- 2

2. Map showing location of hydrologic-data stations, 1979----Back of report

\section{TABLES}

Table 1. Continuous-records (daily discharge) station network, 1979-_.-. 5

2. Crest-stage (peak discharge) station network, 1979--.-12

3. Urban rainfal1-runoff station network, 1979......- 18

4. Active low-flow partial-record stations, 1979----- 21 
PROGRAMS AND ACTIVITIES OF THE MISSOURI DISTRICT,

WATER RESOURCES DIVISION, U.S. GEOLOGICAL SURVEY

FISCAL YEAR 1979

\begin{abstract}
Water-resources investigations of the U.S. Geological Survey in Missouri consist of collecting hydrologic data and conducting interpretive investigations. The data and the results of the investigations are published or released by either the U.S. Geological Survey or by cooperating agencies. This report describes the data-collection activities and investigations in Missouri for the 1979 fiscal year and provides an extensive list of water-resources references for the state.
\end{abstract}

\title{
INTRODUCTION
}

The United States Geological Survey was established on March 3, 1879, in the closing hours of the final session of the 45th Congress; in 1894, a small appropriation was obtained for the specific purpose of "gauging streams and determining the water supply of the United States."

A district office of the Water Resources Division was established in Rolla, Missouri, in 1922, when a cooperative program was begun with the Missouri Bureau of Geology and Mines (now Division of Geology and Land Survey). In cooperation with other Federal and State agencies, the office assesses the quantity and quality of the State's water supply, helps coordinate Federal water-data acquisition activities, collects and distributes information about natural hazards such as floods.

The present functional arrangement within the Missouri district is shown in figure 1.

\section{COOPERATION}

The Missouri district and agencies of the State of Missouri have had cooperative agreements for the systematic collection of streamflow. records since 1921. In fact, about 90 percent of the hydrologic data collected by the Water Resources Division (WRD) in Missouri is in cooperation with local, State, or Federal agencies. 


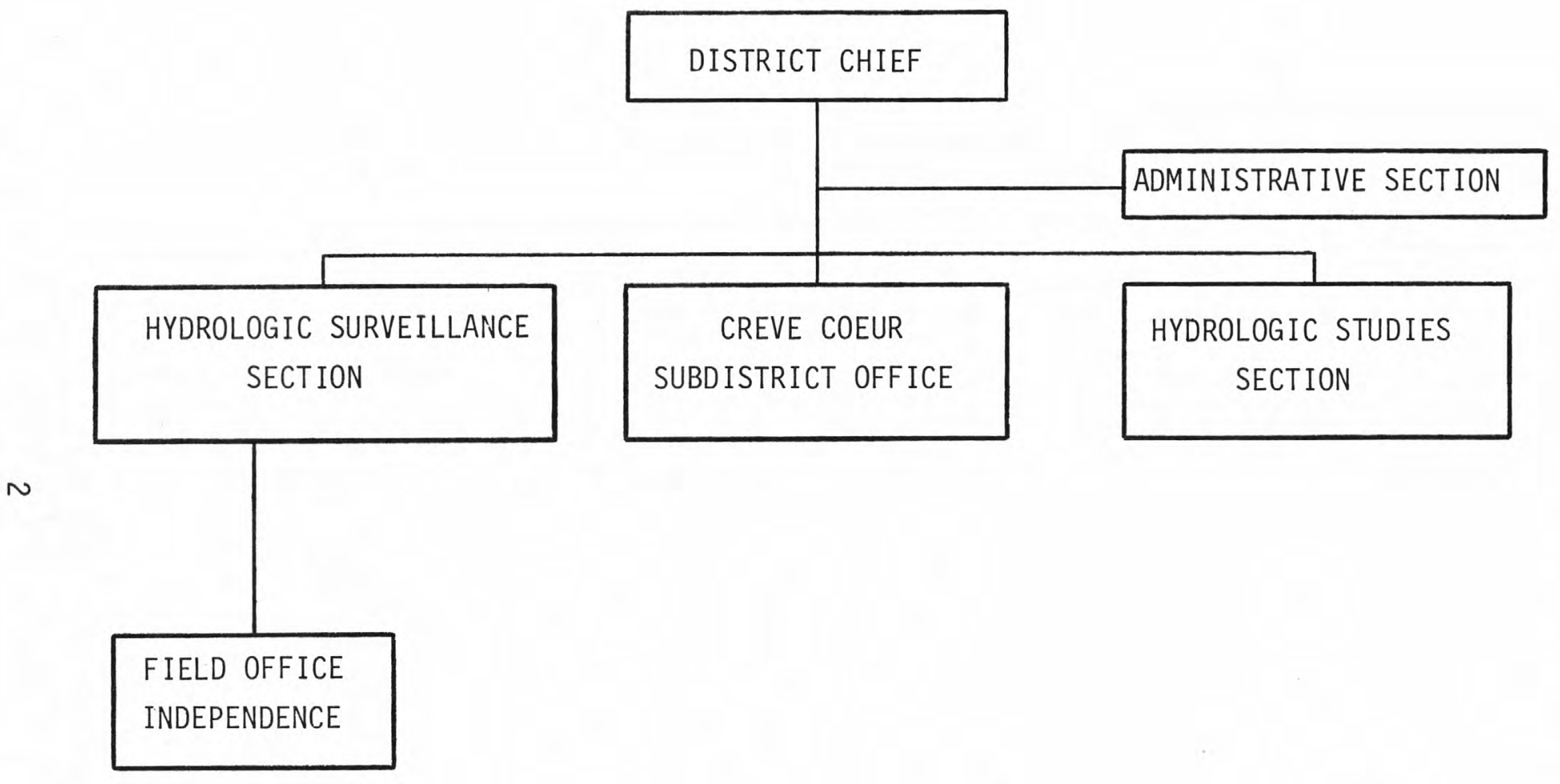

Figure 1.--Organization of the Missouri district. 
The Department of Natural Resources, Division of Geology and Land Survey (formerly Missouri Geological Survey and Water Resources) is the principal State cooperator. Money and services contributed by State agencies are matched by the U.S. Geological Survey with funds authorized specifically for the cooperative program.

The collection of surface-water data by the U.S. Geological Survey began in 1903 in Missouri. These and continuing data are the basis for analytical studies that define streamflow parameters and are ultimately used in water management, planning, and design.

The cooperative collection of ground-water data by the Division of Geology and Land Survey and Water Resources Division, Missouri district, has been continuous since initiation in 0ctober 1963. Many of the published ground-water reports for Missouri are the result of the cooperative program.

The collection of water-quality data is a continuous project that was initiated in 1962 in cooperation with the Missouri Water Pollution Board (now the Department of Natural Resources, Division of Environmental Quality). The objective of the operation of a network of water-quality stations is to provide water-quality data for planning and action programs.

The following are State and local organizations that assist in collecting hydrologic data through cooperative agreements:

Department of Natural Resources

Division of Geology and Land Survey

Division of Environmental Quality, Laboratory Services Program

State Highway Commission

Department of Conservation

St. Louis County, Missouri

Little River Drainage District

City of Springfield

Division of Sanitary Services

City Utilities

The following Federal agencies assist in collection of records by furnishing funds or services:

U.S. Army, Corps of Engineers

St. Louis District

Kansas City District

Little Rock District

Tulsa District

Rock Island District

National Weather Service

Soil Conservation Service

Federal Energy Regulatory Commission (Union Electric Co. of Missouri) 
Hydrologic-data stations are maintained by the Geological Survey at selected key locations throughout the State of Missouri (fig. 2, in the back of report) to constitute a network for obtaining records on stream discharge or stage, reservoir and lake storage, ground-water levels, well and spring discharge, and the quality of surface and ground water. Tables 1-4 are 1istings of hydrologic-data stations, in downstream order, and source of funding.

\section{MISSOURI DISTRICT PROJECTS}

The Missouri district projects address a wide range of hydrologic needs. Projects are designed and planned with the cooperators to be responsive to the needs of people in Missouri. The following pages give information on all projects now in progress and anticipated or expanded work for the future.

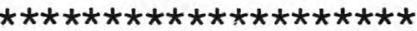

PROJECT TITLE: Collection of surface-water data M 00-001

COOPERATORS: Department of Natural Resources

Division of Geology and Land Survey

Division of Environmental Quality, Laboratory

Services Program

State Highway Commission

Department of Conservation

U.S. Army, Corps of Engineers

National Weather Service

Union Electric Company of Missouri

Little River Drainage District

City of Springfield Utilities

City of Springfield, Division of Sanitary Services

LOCATION: $\quad$ Statewide

PROJECT CHIEF: M. E. Janson

PROBLEM: $\quad$ Surface-water information is needed for purposes of surveillance, planning design, hazard warning, and operation and management in water-related fields such as water supply, hydroelectric power, flood control, irrigation, bridge and culvert design, wildlife management, pollution abatement, flood-plain management, and water resources development. 
Table 1.--Continuous-records (daily discharge) station network, 1979

[DG\&LS, Division of Geology and Land Survey; City of Spfd., City of Springfield; Mo. Highway Comm., Missouri Highway Commission; Mo. Conserv. Comm., Missouri Conservation Commission; USGS Coop, U.S. Geological Survey Coop; USGS Fed., U.S. Geological

Survey-Federal; CE RI, Corps of Engineers, Rock Island; CE STL, Corps of Engineers, St. Louis; CE KC, Corps of Engineers, Kansas City; CE TU, Corps of Engineers, Tulsa; CE LR, Corps of Engineers, Little Rock; UE, Union Electric]

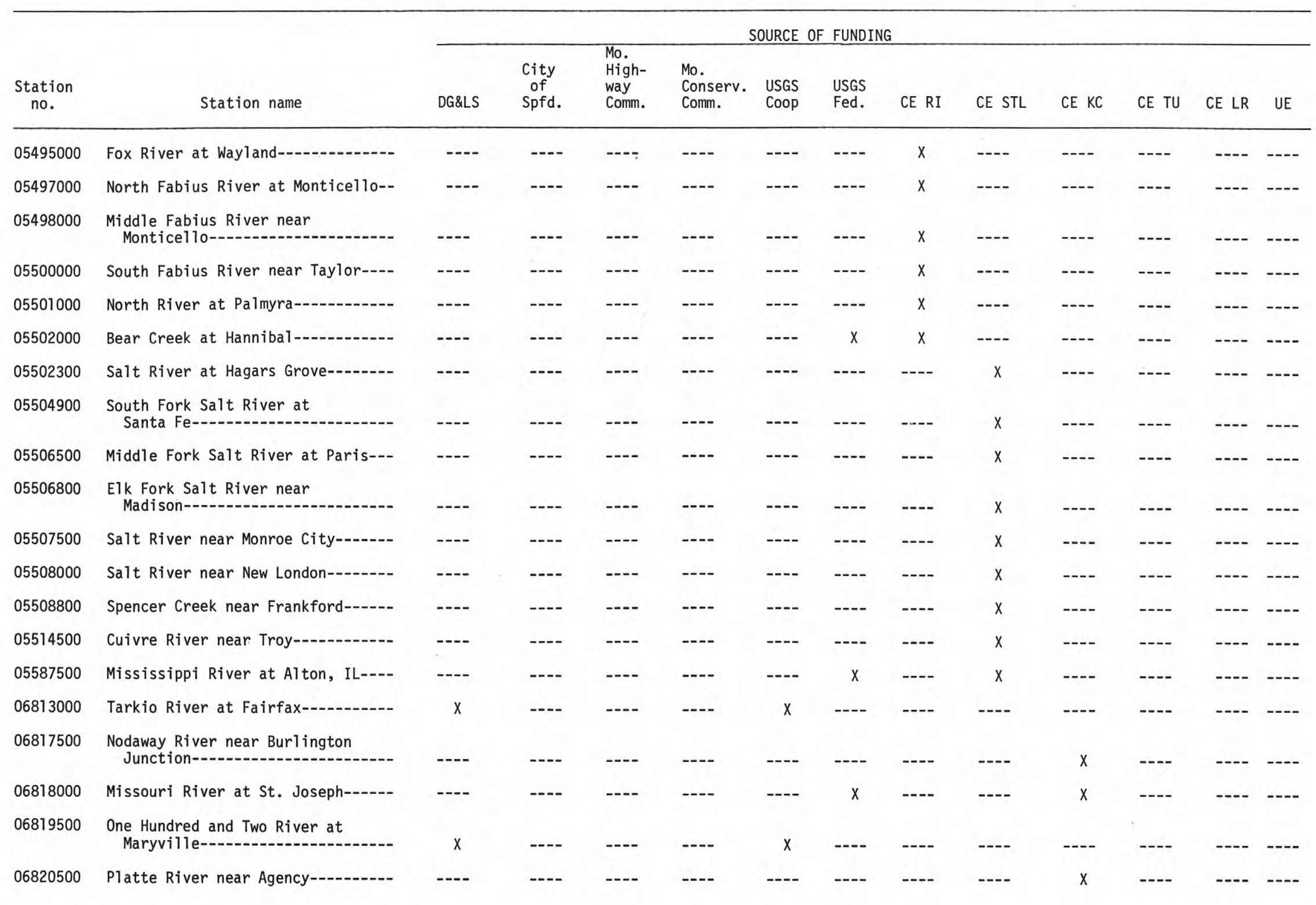


Table 1.--Continuous-records (daily discharge) station network, 1979--continued

[DG\&LS, Division of Geology and Land Survey; City of Spfd., City of Springfield; Mo. Highway Comm., Missouri Highway Commission; Mo. Conserv. Comm., Missouri Conservation Commission; USGS Coop, U.S. Geological Survey Coop; USGS Fed, U.S. Geological Survey-Federal; CE RI, Corps of Engineers, Rock Island; CE STL, Corps of Engineers, St. Louis; CE KC, Corps of Engineers, Kansas City; CE TU, Corps of Engineers, Tulsa; CE LR, Corps of Engineers, Little Rock; UE, Union Electric]

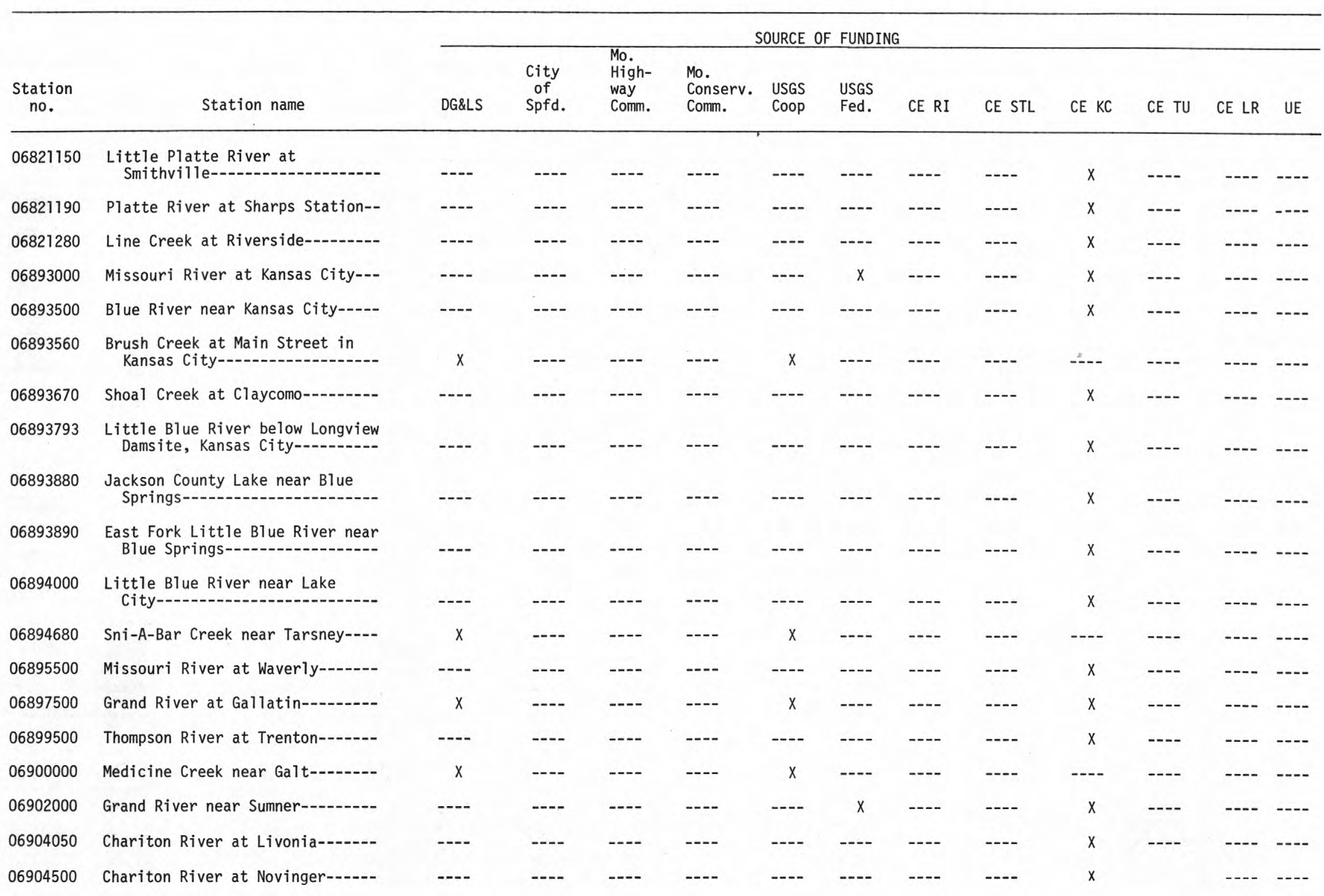


Table 1.--Continuous-records (daily discharge) station network, 1979--continued

[DG\&LS, Division of Geology and Land Survey; City of Spfd., City of Springfield; Mo. Highway Comm., Missouri Highway

Commission; Mo. Conserv. Comm., Missouri Conservation Commission; USGS Coop, U.S. Geological Survey Coop; USGS Fed., U.S. Geological Survey-Federal; CE RI, Corps of Engineers, Rock Island; CE STL, Corps of Engineers, St. Louis; CE KC, Corps of Engineers, Kansas City; CE TU, Corps of Engineers, Tulsa; CE LR, Corps of Engineers, Little Rock; UE, Union Electric]

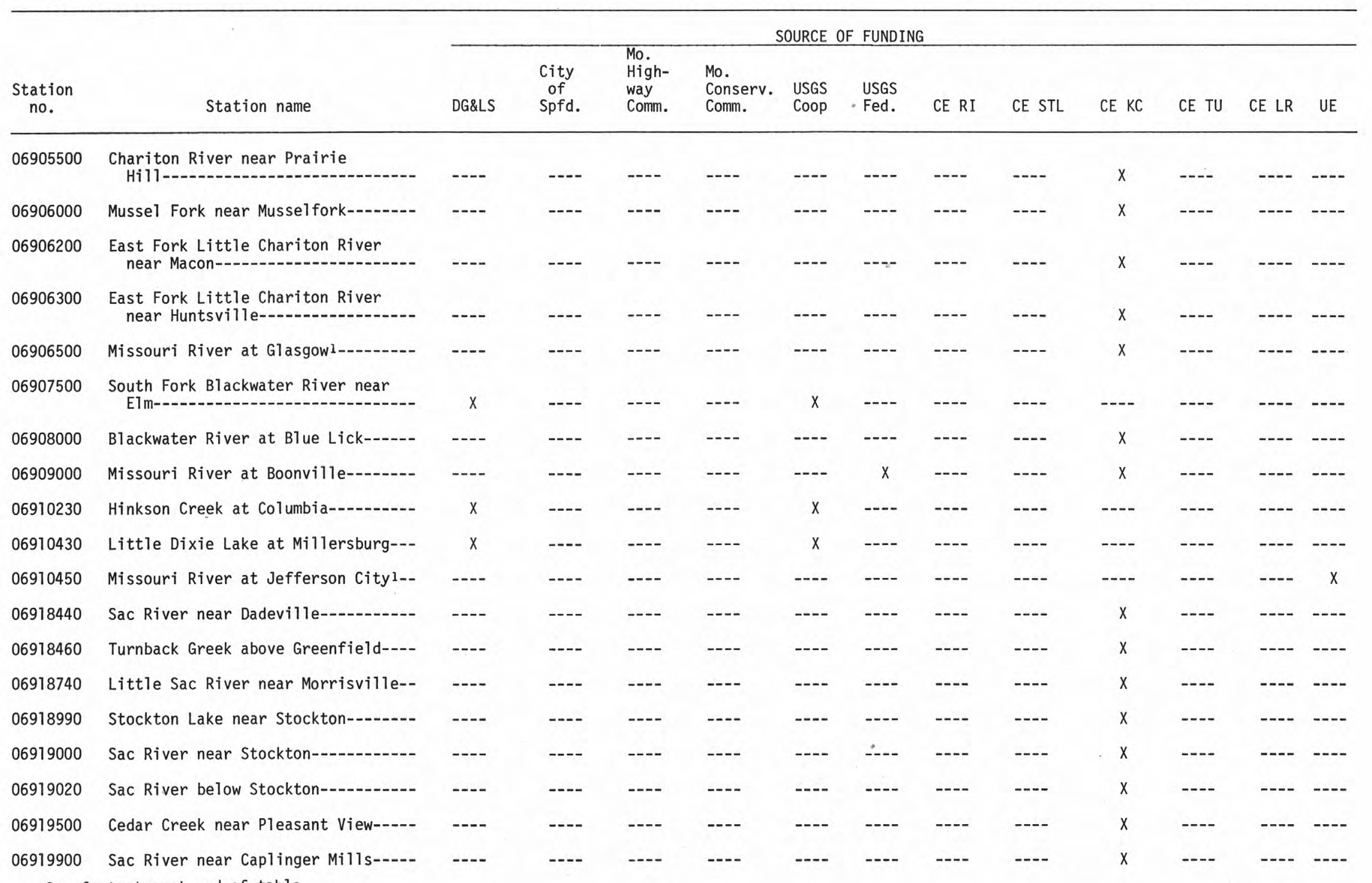


Table 1.--Continuous-records (daily discharge) station network, 1979--continued

[DG\&LS, Division of Geology and Land Survey; City of Spfd., City of Springfield; Mo. Highway Comm., Missouri Highway Commission; Mo. Conserv. Comm., Missouri Conservation Commission; USGS Coop, U.S. Geological Survey Coop; USGS Fed., U.S. Geological

Survey-Federa1; CE RI, Corps of Engineers, Rock Island; CE STL, Corps of Engineers, St. Louis; CE KC, Corps of Engineers, Kansas City; CE TU, Corps of Engineers, Tulsa; CE LR, Corps of Engineers, Little Rock; UE, Union Electric]

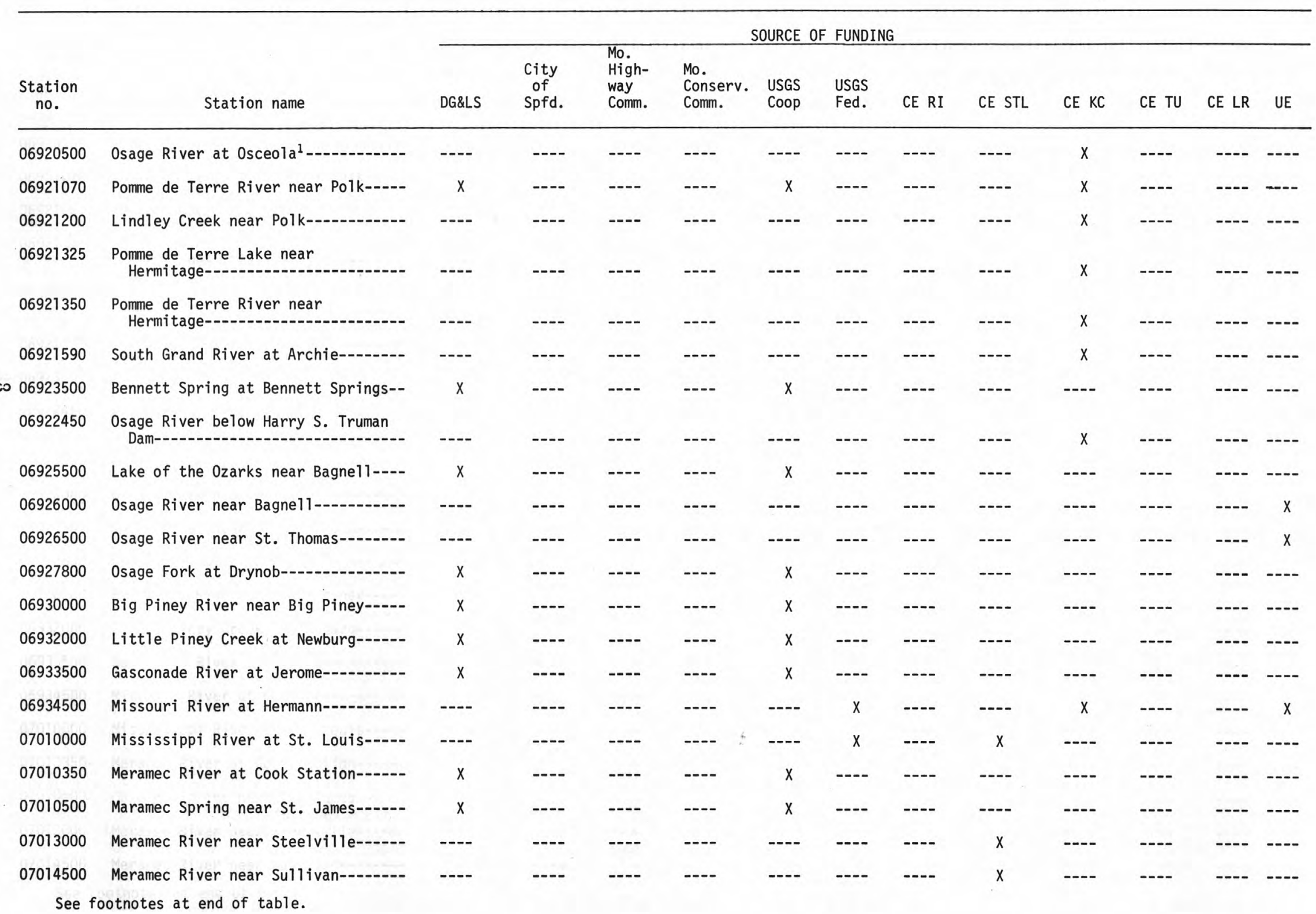


Table 1.--Continuous-records (daily discharge) station network, 1979--continued

[DG\&LS, Division of Geology and Land Survey; City of Spfd., City of Springfield; Mo. Highway Comm., Missouri Highway

Commission; Mo. Conserv. Comm., Missouri Conservation Commission; USGS Coop, U.S. Geological Survey Coop; USGS Fed., U.S. Geological Survey-Federal; CE RI, Corps of Engineers, Rock Island; CE STL, Corps of Engineers, St. Louis; CE KC, Corps of Engineers, Kansas City; CE TU, Corps of Engineers, Tulsa; CE LR, Corps of Engineers, Little Rock; UE, Union Electric]

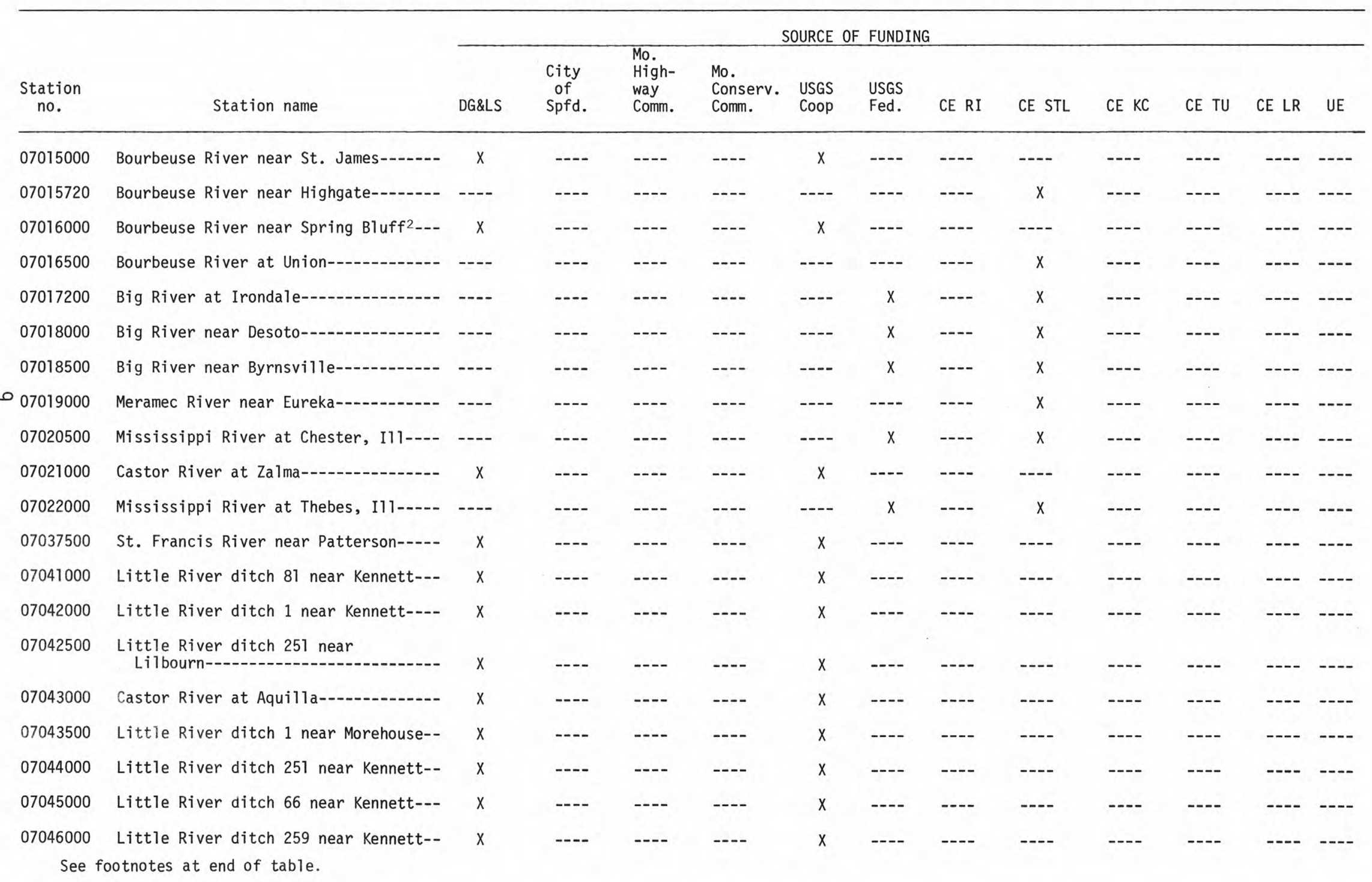


Table 1.--Continuous-records (daily discharge) station network, 1979--continued

[DG\&LS, Division of Geology and Land Survey; City of Spfd., City of Springfield; Mo. Highway Comm., Missouri Highway Commission; Mo. Conserv. Comm., Missouri Conservation Commission; USGS Coop, U.S. Geological Survey Coop; USGS Fed., U.S. Geological Survey-Federal; CE RI, Corps of Engineers, Rock Island; CE STL, Corps of Engineers, St. Louis; CE KC, Corps of Engineers, Kansas City; CE TU, Corps of Engineer,s, Tulsa; CE LR, Corps of Engineers, Little Rock; UE, Union Electric]

\begin{tabular}{|c|c|c|c|c|c|c|c|c|c|c|c|c|c|}
\hline \multirow[b]{2}{*}{$\begin{array}{l}\text { Station } \\
\text { no. }\end{array}$} & \multirow[b]{2}{*}{ Station name } & \multicolumn{11}{|c|}{ SOURCE OF FUNDING } & \multirow[b]{2}{*}{ UE. } \\
\hline & & DG\&LS & $\begin{array}{c}\text { City } \\
\text { of } \\
\text { Spfd. }\end{array}$ & $\begin{array}{l}\text { Mo. } \\
\text { High- } \\
\text { way } \\
\text { Comm. }\end{array}$ & $\begin{array}{l}\text { Mo. } \\
\text { Conserv. } \\
\text { Comm. }\end{array}$ & $\begin{array}{l}\text { USGS } \\
\text { Coop }\end{array}$ & $\begin{array}{l}\text { USGS } \\
\text { Fed. }\end{array}$ & CE RI & CE STL & CE KC & CE TU & CE LR & \\
\hline 07050580 & James River near Strafford------- & --- & --- & --- & --- & --- & $x$ & --- & --- & --- & --- & $x$ & --- \\
\hline 07050700 & James River near Springfield--.-- & $x$ & --- & --- & --- & $x$ & --- & --- & --- & --- & --- & --- & --- \\
\hline 07050750 & James River near Nixa ${ }^{3}$ & $x$ & --- & --- & --- & $x$ & --- & --- & --- & --- & --- & --- & ---- \\
\hline 07052100 & Wilsons Creek near Springfield-...-- & --- & $x$ & $-\cdots$ & --- & $x$ & ---- & --- & --- & --- & --- & --- & --- \\
\hline 07052160 & Wilsons Creek near Battlefield------- & --- & $x$ & ---- & ---- & $x$ & ---- & ---- & --- & --- & --- & --- & --- \\
\hline 07052250 & James River near Boaz-...- & --- & $x$ & --- & --- & $x$ & --- & --- & --- & --- & --- & $-\cdots$ & --- \\
\hline 07052500 & James River at Galena-..- & --- & --- & $-\cdots$ & --- & --- & --- & --- & --- & --- & --- & $x$ & --- \\
\hline 07053400 & Table Rock Lake near Branson ${ }^{1}$ & $x$ & --- & --- & --- & $x$ & ---- & --- & --- & --- & --- & $x$ & --- \\
\hline 07053500 & White River near Branson ${ }^{1}$ & & ORDS FU & SHED BY & ORPS OF EN & GINEERS & & & & & & & \\
\hline 07054000 & White River at Forsyth ${ }^{1}$ & $x$ & --- & --- & --- & $x$ & --- & --- & --- & --- & --- & $x$ & --- \\
\hline 07057500 & North Fork River near Tecumseh--.--- & $-\cdots$ & --- & --- & $-\cdots$ & --- & --- & --- & $-\cdots$ & --- & --- & $x$ & --- \\
\hline 07058000 & Bryant Creek near Tecumseh-.......-- & --- & --- & --- & --- & --- & --- & --- & --- & --- & --- & $x$ & --- \\
\hline 07061300 & $\begin{array}{l}\text { East Fork Black River at } \\
\text { Lesterville-1 }\end{array}$ & $x$ & --- & $-\cdots$ & --- & $x$ & --- & --- & --- & --- & --- & $-\cdots$ & --- \\
\hline 07061500 & Black River near Annapolis------ & --- & --- & $-\cdots$ & $-\cdots$ & --- & --- & --- & --- & --- & --- & $x$ & ---- \\
\hline 07062000 & Clearwater Lake near Piedmont-..---- & --- & --- & --- & --- & --- & ---- & --- & --- & --- & --- & $x$ & --- \\
\hline 07062500 & Black River at Leeper. & --- & --- & --- & --- & --- & --- & --- & --- & --- & --- & $x$ & --- \\
\hline 07063000 & Black River at Poplar Bluff-...-- & --- & --- & $-\cdots$ & --- & --- & --- & --- & $-\cdots$ & --- & --- & $x$ & --- \\
\hline 07063250 & Black River at Gillis Bluff ${ }^{4}$ & --- & --- & ---- & --- & --- & --- & --- & --- & --- & $-\cdots$ & $x$ & --- \\
\hline 07063500 & Cane Creek near Harviel1 $1^{4}$ & --- & --- & --- & --- & --- & --- & --- & --- & --- & --- & $x$ & $-\cdots$ \\
\hline 07065000 & Round Spring at Round Spring--.--- & $x$ & --- & --- & ---- & $x$ & --- & --- & --- & --- & --- & --- & --- \\
\hline 07065500 & Alley Spring at Alley- & $x$ & --- & $-\cdots$ & --- & $x$ & --- & --- & --- & --- & --- & --- & --- \\
\hline 07066000 & Jacks Fork at Eminence-1 & $x$ & --- & --- & --- & $x$ & --- & --- & --- & $-\cdots$ & --- & --- & --- \\
\hline $\begin{array}{r}07067000 \\
\text { See }\end{array}$ & $\begin{array}{l}\text { Current River at Van Buren-1- } \\
\text { ootnotes at end of table. }\end{array}$ & $x$ & ---- & --- & --- & $X$ & ---- & --- & --- & --- & ---- & --- & --- \\
\hline
\end{tabular}


Table 1.--Continuous-records (daily discharge) station network, 1979--continued

[DG\&LS, Division of Geology and Land Survey; City of Spfd., City of Springfield; Mo. Highway Comm., Missouri Highway Commission; Mo. Conserv. Comm., Missouri Conservation Commission; USGS Coop, U.S. Geological Survey Coop; USGS Fed., U.S. Geological Survey-Federa1; CE RI, Corps of Engineers, Rock Island; CE STL, Corps of Engineers, St. Louis; CE KC, Corps of Engineers, Kansas City; CE TU, Corps of Engineers, Tulsa; CE LR, Corps of Engineers, Little Rock, UE, Union Electric]

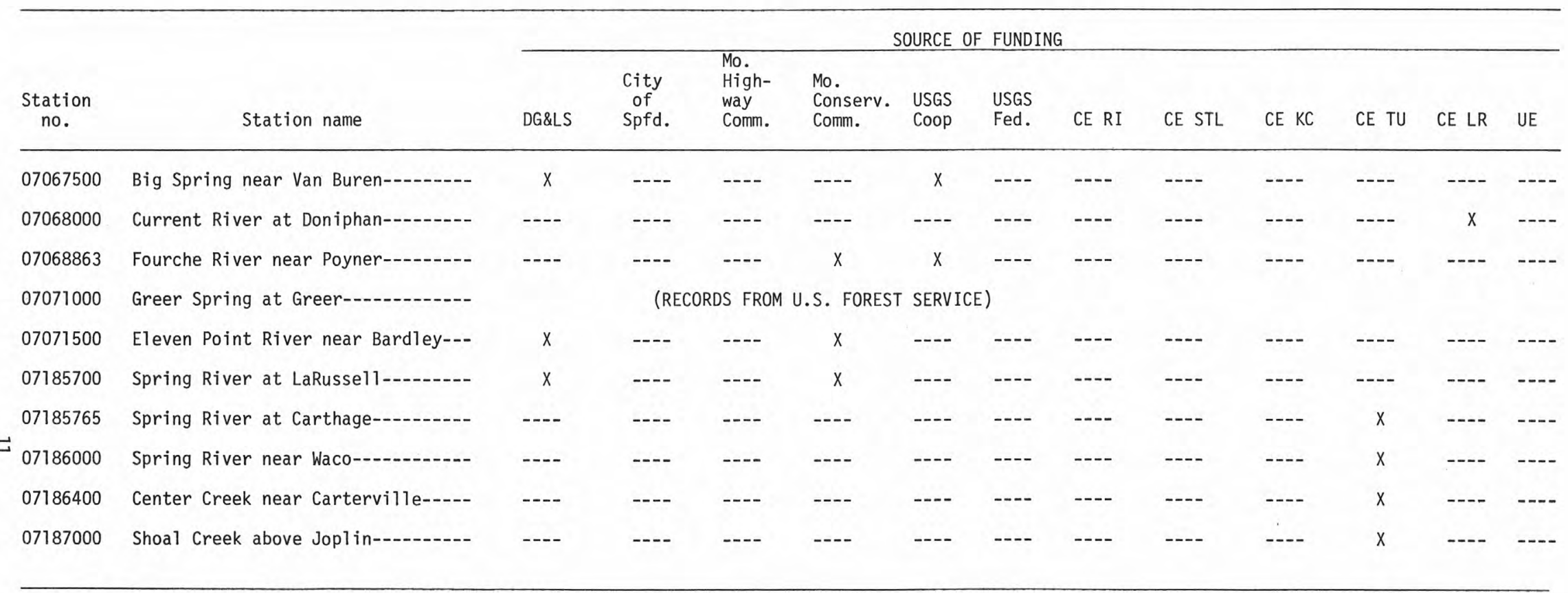

\footnotetext{
${ }^{1}$ Stage only.

2Discharge below $1,000 \mathrm{ft}^{3} / \mathrm{s}$ not computed.

3 Discharge above $100 \mathrm{ft}^{3} / \mathrm{s}$ not computed.

"Stage-discharge relation only.
} 
Table 2.--Crest-stage (i)eak discharge) station network, 1979

[DG\&LS, Division of Geology and Land Survey; City of Spfd., City of Springfield; Mo. Highway Comm., Missouri Highway Commission; Mo. Conserv. Comm., Missouri Conservation Commission; USGS Coop, U.S. Geological Survey Coop; USGS Fed., U.S. Geological

Survey-Federal; CE RI, Corps of Engineers, Rock Island; CE STL, Corps of Engineers, St. Louis; CE KC, Corps of Engineers, Kansas City; CE TU, Corps of Engineers, Tulsa; CE LR, Corps of Engineers, Little Rock; UE, Union Electric]

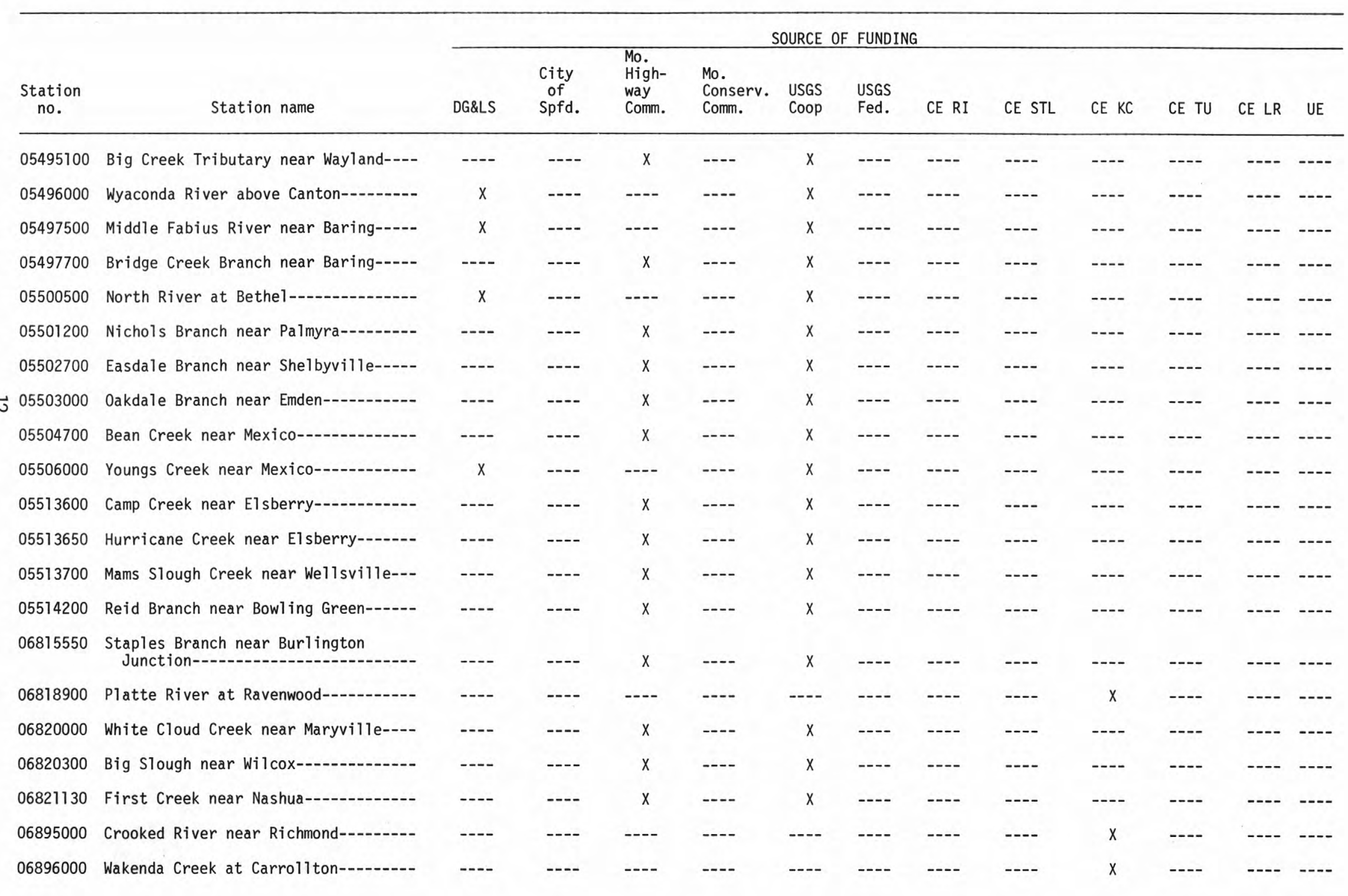


Table 2.--Crest-stage (peak discharge) station network, 1979--continued

[DG\&LS, Division of Geology and Land Survey; City of Spfd., City of Springfield; Mo. Highway Comm., Missouri Highway Commission; Mo. Conserv. Comm., Missouri Conservation Commission; USGS Coop, U.S. Geological Survey Coop; USGS Fed., U.S. Geological

Survey-Federal; CE RI, Corps of Engineers, Rock Island; CE STL, Corps of Engineers, St. Louis; CE KC, Corps of Engineers, Kansas City; CE TU, Corps of Engineers, Tulsa; CE LR, Corps of Engineers, Little Rock; UE, Union Electric]

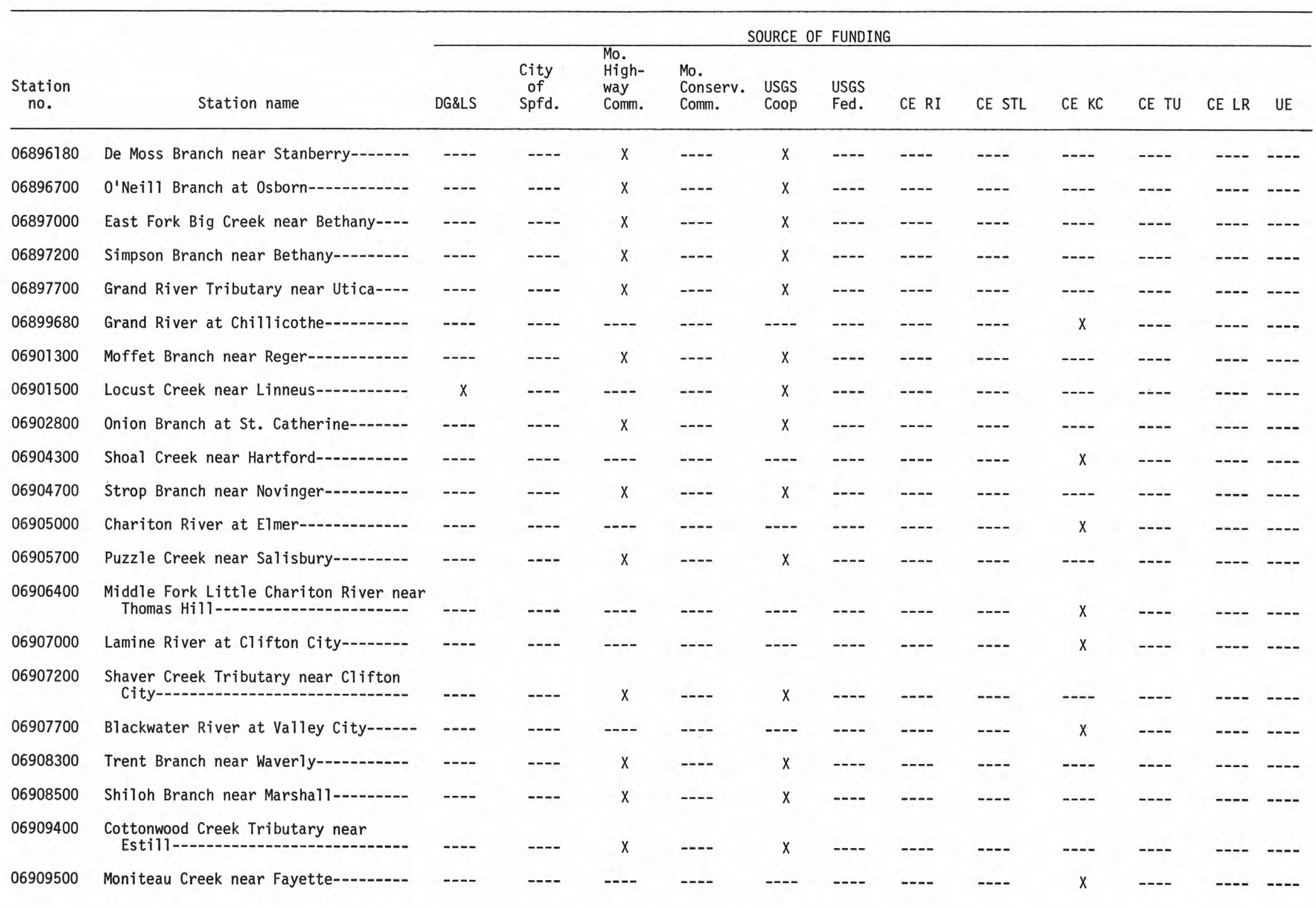


Table 2.--Crest-stage (peak discharge) station network, 1979--continued

[DG\&LS, Division of Geology and Land Survey; City of Spfd., City of Springfield; Mo. Highway Comm., Missouri Highway Commission; Mo. Conserv. Comm., Missouri Conservation Commission; USGS Coop, U.S. Geological Survey Coop; USGS Fed., U.S. Geological Survey-Federa 1; CE RI, Corps of Engineers, Rock Island; CE STL, Corps of Engineers, St. Louis; CE KC, Corps of Engineers, Kansas City; CE TU, Corps of Engineers, Tulsa; CE LR, Corps of Engineers, Little Rock; UE, Union Electric]

\begin{tabular}{|c|c|c|c|c|c|c|c|c|c|c|c|c|c|}
\hline \multirow[b]{2}{*}{$\begin{array}{l}\text { Station } \\
\text { no. }\end{array}$} & \multirow[b]{2}{*}{ Station name } & \multicolumn{12}{|c|}{ SOURCE OF FUNDING } \\
\hline & & DG\&LS & $\begin{array}{l}\text { City } \\
\text { of } \\
\text { Spfd. }\end{array}$ & $\begin{array}{l}\text { High- } \\
\text { way } \\
\text { Comm. }\end{array}$ & $\begin{array}{l}\text { Mo. } \\
\text { Conserv. } \\
\text { Comm. }\end{array}$ & $\begin{array}{l}\text { USGS } \\
\text { Coop }\end{array}$ & $\begin{array}{l}\text { USGS } \\
\text { Fed. }\end{array}$ & CE RI & CE STL & CE KC & CE TU & CE LR & UE \\
\hline 06909700 & $\begin{array}{l}\text { Petite Saline Creek Tributary near } \\
\text { Bellair- }\end{array}$ & --- & --- & $x$ & --- & $x$ & --- & --- & --- & --- & --- & --- & --- \\
\hline 06910200 & Cow Branch near Columbia--------- & --- & --- & $x$ & --- & $x$ & ---- & --- & --- & --- & --- & --- & --- \\
\hline 06910250 & Traxler Branch near Columbia-------- & --- & --- & $x$ & --- & $x$ & --- & --- & --- & --- & --- & --- & --- \\
\hline 06910400 & Baldwin Branch near Jefferson City--- & --- & --- & $x$ & --- & $x$ & ---- & --- & --- & --- & --- & --- & --- \\
\hline 06910410 & Cedar Creek near Columbia--- & $x$ & --- & --- & --- & $x$ & --- & --- & $-\cdots$ & --- & --- & --- & --- \\
\hline 06910430 & $\begin{array}{l}\text { Dickerson Creek Tributary near } \\
\text { Jefferson City- }\end{array}$ & --- & --- & $x$ & --- & $x$ & --- & --- & $-\cdots$ & --- & --- & --- & --- \\
\hline 06910500 & Moreau River near Jefferson City----- & --- & --- & --- & --- & --- & --- & --- & --- & $x$ & --- & --- & --- \\
\hline 06910700 & $\begin{array}{l}\text { Hazel Branch Tributary near } \\
\text { Wardsville-- }\end{array}$ & --- & --- & $x$ & --- & $x$ & --- & ---- & --- & --- & --- & --- & --- \\
\hline 06918200 & $\begin{array}{l}\text { North Fork Panther Tributary near } \\
\text { Appleton City }\end{array}$ & --- & --- & $x$ & --- & $x$ & --- & --- & --- & --- & --- & --- & --- \\
\hline 06918300 & $\begin{array}{l}\text { West Fork Clear Creek Tributary near } \\
\text { Nevada- }\end{array}$ & --- & $-\cdots$ & $x$ & --- & $x$ & --- & ---- & --- & --- & ---- & --- & ---- \\
\hline 06918400 & $\begin{array}{l}\text { Pickerel Creek Tributary near } \\
\text { Republic- }\end{array}$ & --- & $-\cdots$ & $x$ & --- & $x$ & --- & --- & --- & --- & --- & --- & --- \\
\hline $069 \cdot 18750$ & Franco Branch near Brighton--.---- & --- & --- & $x$ & ---- & $x$ & --- & --- & --- & --- & --- & --- & --- \\
\hline 06919200 & $\begin{array}{l}\text { Sac River Tributary near Caplinger } \\
\text { Mills }\end{array}$ & --- & --- & $x$ & --- & $x$ & --- & --- & --- & --- & --- & --- & --- \\
\hline 06920800 & Big Muddy Creek at Lowry City-.---- & $-\cdots$ & --- & $x$ & --- & $x$ & --- & --- & --- & --- & --- & --- & --- \\
\hline 06921100 & 01 inger Creek near Buffalo-_...-.- & --- & --- & $x$ & --- & $x$ & --- & --- & --- & --- & --- & --- & --- \\
\hline 06921400 & Ferguson Branch at Nemo- & --- & --- & $x$ & --- & $x$ & --- & --- & --- & --- & --- & --- & --- \\
\hline 06921720 & Big Creek at Blairstown-- & --- & --- & ---- & ---- & --- & --- & --- & --- & $x$ & --- & --- & $-\cdots$ \\
\hline 06921800 & Grandaddy Creek near Urich-_- & --- & --- & $x$ & --- & $x$ & $-\cdots$ & --- & $-\cdots$ & --- & --- & --- & --- \\
\hline 06922600 & $\begin{array}{l}\text { Little Turkey Creek Tributary near } \\
\text { Warsaw- }\end{array}$ & --- & --- & $x$ & --- & $x$ & --- & --- & --- & --- & --- & --- & --- \\
\hline
\end{tabular}


Table 2.--Crest-stage (peak discharge) station network, 1979--continued

[DG\&LS, Division of Geology and Land Survey; City of Spfd., City of Springfield; Mo. Highway Comm., Missouri Highway Commission; Mo. Conserv. Comm., Missouri Conservation Commission; USGS Coop, U.S. Geological Survey Coop; USGS Fed., U.S. Geological Survey-Federal; CE RI, Corps of Engineers, Rock Island; CE STL, Corps of Engineers, St. Louis; CE KC, Corps of Engineers, Kansas City; CE TU, Corps of Engineers, Tulsa; CE LR, Corps of Engineers, Little Rock; UE, Union Electric]

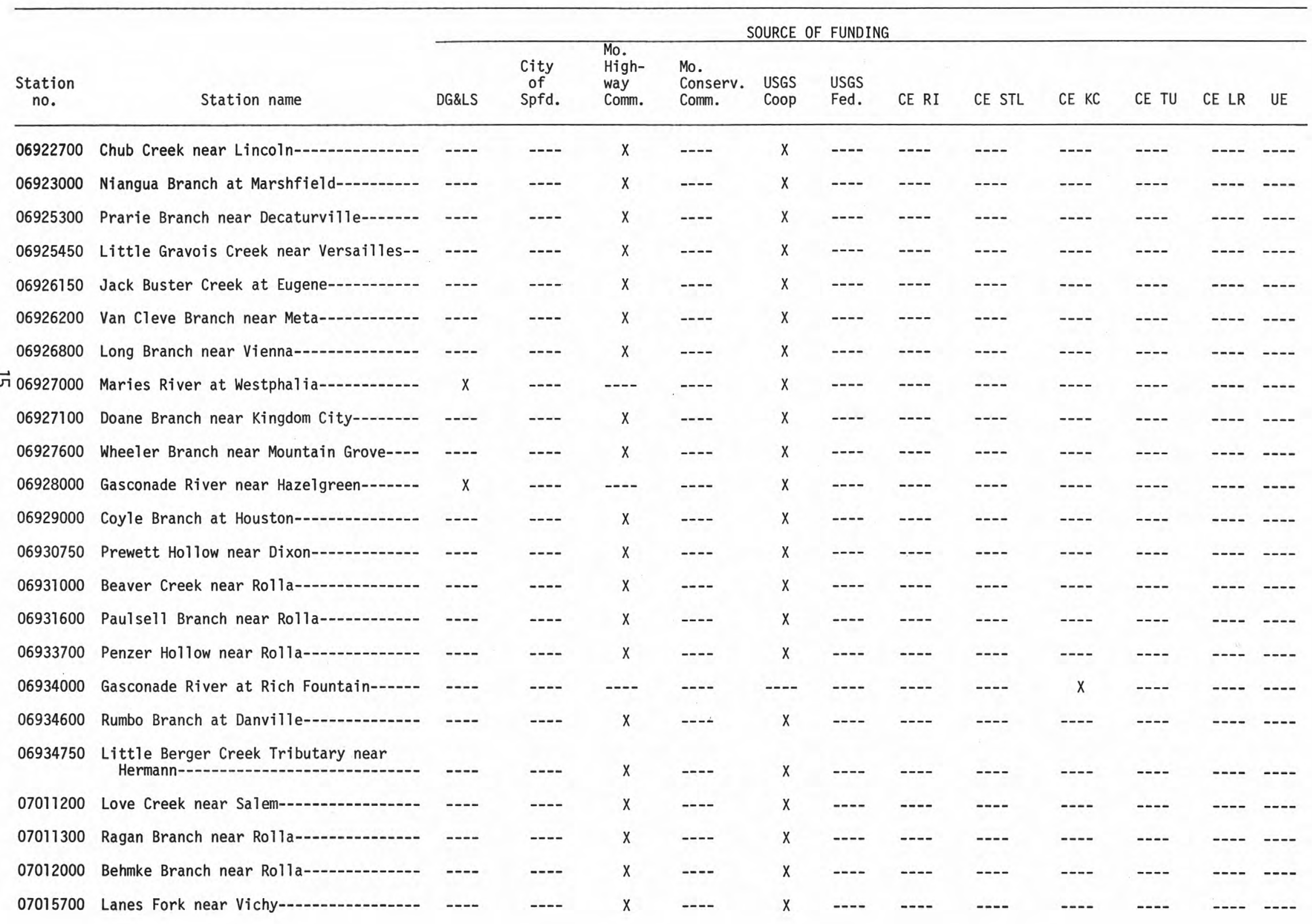


Table 2.--Crest-stage (peak discharge) station network, 1979--continued

[DG\&LS, Division of Geology and Land Survey; City of Spfd., City of Springfield; Mo. Highway Comm., Missouri Highway Commission; Mo. Conserv. Comm., Missouri Conservation Commission; USGS Coop, U.S. Geological Survey Coop; USGS Fed., U.S. Geological Survey-Federal; CE RI, Corps of Engineers, Rock Island; CE STL, Corps of Engineers, St. Louis; CE KC, Corps of Engineers, Kansas City; CE TU, Corps of Engineers, Tulsa; CE LR, Corps of Engineers, Little Rock; UE, Union Electric]

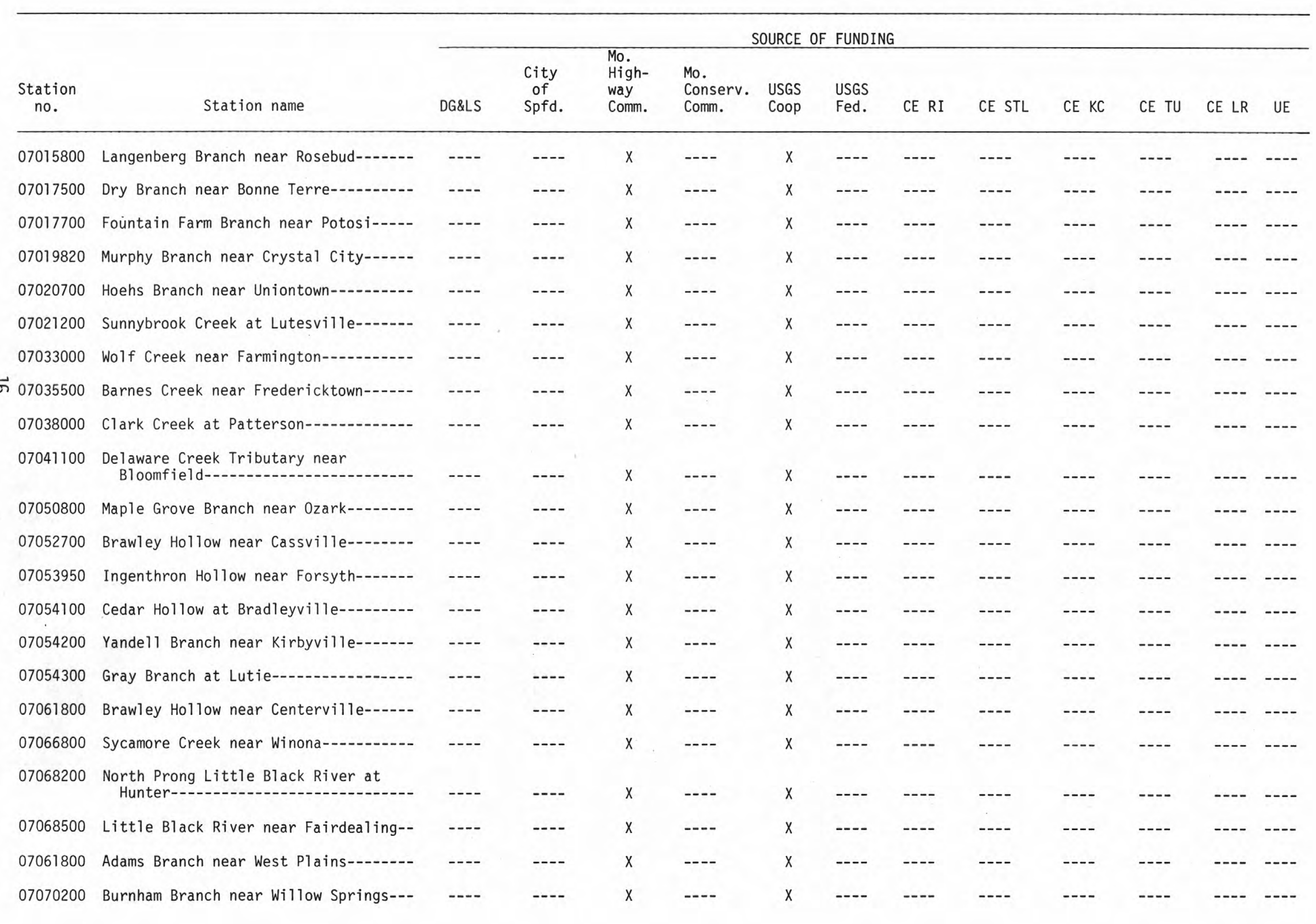


Table 2.--Crest-stage (peak discharge) station network, 1979--continued

[DG\&LS, Division of Geology and Land Survey; City of Spfd., City of Springfield; Mo. Highway Comm., Missouri Highway Commission; Mo. Conserv. Comm., Missouri Conservation Commission; USGS Coop, U.S. Geological Survey Coop; USGS Fed., U.S. Geological Survey-Federal; CE RI, Corps of Engineers, Rock Island; CE STL, Corps of Engineers, St. Louis; CE KC, Corps of Engineers, Kansas City; CE TU, Corps of Engineers, Tulsa; CE LR, Corps of Engineers, Little Rock; UE, Union Electric]

\begin{tabular}{|c|c|c|c|c|c|c|c|c|c|c|c|c|c|}
\hline \multirow[b]{2}{*}{$\begin{array}{c}\text { Station } \\
\text { no. }\end{array}$} & \multirow[b]{2}{*}{ Station name } & \multicolumn{11}{|c|}{ SOURCE OF FUNDING } & \multirow[b]{2}{*}{ UE } \\
\hline & & DG\&LS & $\begin{array}{l}\text { City } \\
\text { of } \\
\text { Spfd. }\end{array}$ & $\begin{array}{l}\text { High- } \\
\text { way } \\
\text { Comm. }\end{array}$ & $\begin{array}{l}\text { Mo. } \\
\text { Conserv. } \\
\text { Comm. }\end{array}$ & $\begin{array}{l}\text { USGS } \\
\text { Coop }\end{array}$ & $\begin{array}{l}\text { USGS } \\
\text { Fed. }\end{array}$ & CE RI & CE STL & CE KC & CE TU & CE LR & \\
\hline 07071800 & Williams Spring Branch near Alton---- & --- & ---- & $x$ & ---- & $x$ & ---- & ---- & ---- & ---- & ---- & ---- & ---- \\
\hline 07185600 & South Fork Stahl Creek near Miller--- & --- & ---- & $x$ & ---- & $x$ & --- & --- & --- & ---- & --- & --- & $-\cdots$ \\
\hline 07186950 & North Fork Carver Branch at Diamond-- & --- & --- & $x$ & ---- & $x$ & ---- & ---- & ---- & ---- & ---- & --- & --- \\
\hline
\end{tabular}


Table 3.--Urban rainfall-runoff station network, 1979

[DG\&LS, Division of Geology and Land Survey; City of Spfd., City of Springfield; Mo. Highway Comm., Missouri Highway Commission; St. Louis Co., St. Louis County; USGS Coop, U.S. Geological Survey Coop; USGS Fed., U.S. Geological

Survey-Federal; CE RI, Corps of Engineers, Rock Island; CE STL, Corps of Engineers, St. Louis; CE KC, Corps of Engineers, Kansas City; CE TU, Corps of Engineers, Tulsa; CE LR, Corps of Engineers, Little Rock; UE, Union Electric]

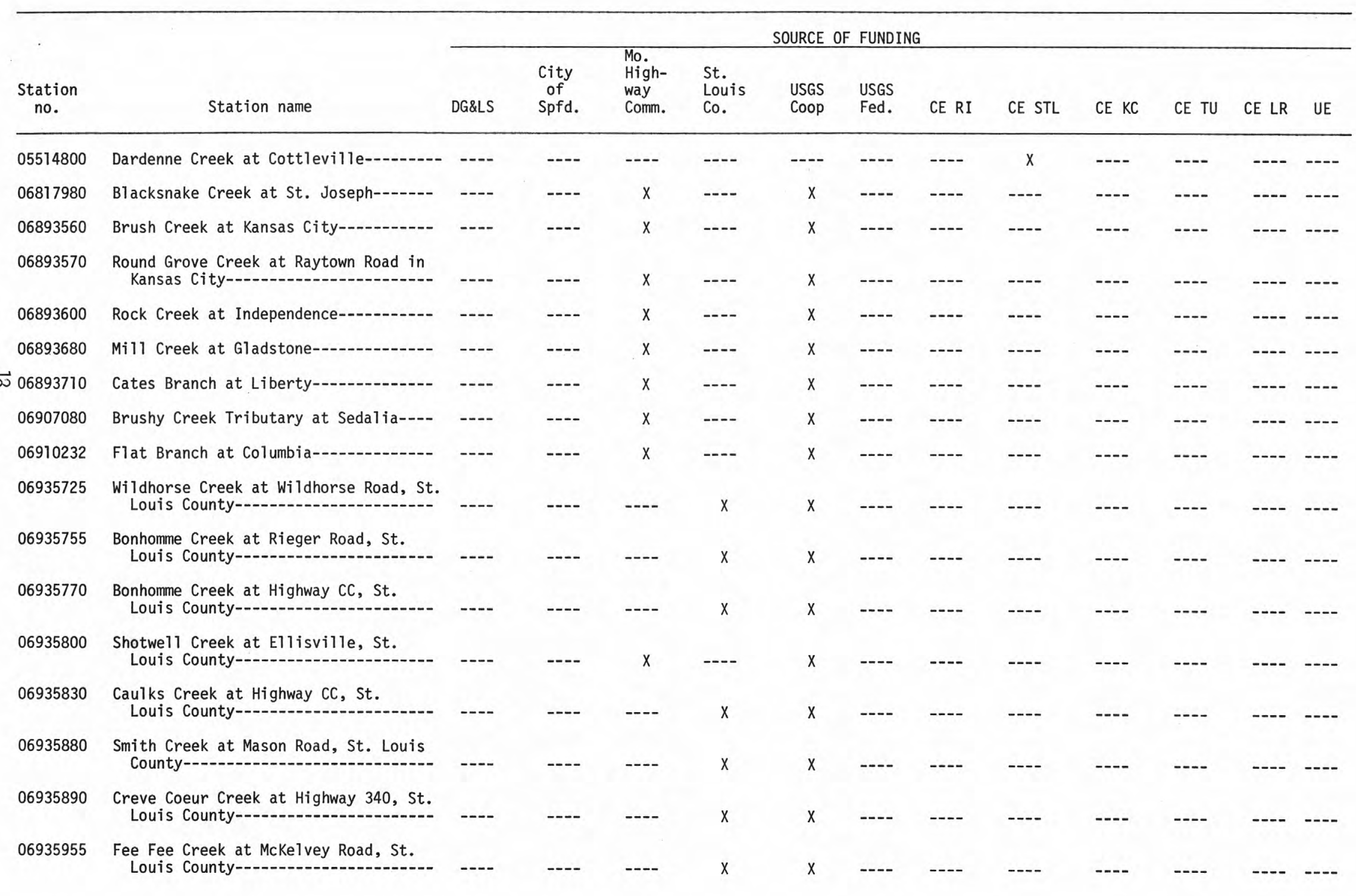


Table 3.--Urban rainfall-runoff station network, 1979--continued

[DG\&LS, Division of Geology and Land Survey; City of Spfd., City of Springfield; Mo. Highway Comm., Missouri Highway

Commission; St. Louis Co. St. Louis County; USGS Coop, U.S. Geological Survey Coop; USGS Fed., U.S. Geological

Survey-Federal; CE RI, Corps of Engineers, Rock Island; CE STL, Corps of Engineers, St. Louis; CE KC, Corps of Engineers, Kansas City; CE TU, Corps of Engineers, Tulsa; CE LR, Corps of Engineers, Little Rock; UE, Union Electric]

\begin{tabular}{|c|c|c|c|c|c|c|c|c|c|c|c|c|c|}
\hline \multirow[b]{2}{*}{$\begin{array}{l}\text { Station } \\
\text { no. }\end{array}$} & \multirow[b]{2}{*}{ Station name } & \multicolumn{11}{|c|}{ SOURCE OF FUNDING } & \multirow[b]{2}{*}{ UE } \\
\hline & & DG\&LS & $\begin{array}{l}\text { City } \\
\text { of } \\
\text { Spfd. }\end{array}$ & $\begin{array}{l}\text { High- } \\
\text { way } \\
\text { Comm. }\end{array}$ & $\begin{array}{l}\text { St. } \\
\text { Louis } \\
\text { Co. }\end{array}$ & $\begin{array}{l}\text { USGS } \\
\text { Coop }\end{array}$ & $\begin{array}{l}\text { USGS } \\
\text { Fed. }\end{array}$ & CE RI & CE STL & CE KC & CE TU & CE LR & \\
\hline 06935980 & $\begin{array}{l}\text { Cowmire Creek at Kirchner Road, St. } \\
\text { Louis County- }\end{array}$ & --- & --- & --- & $x$ & $x$ & --- & --- & --- & $-\cdots$ & --- & --- & --- \\
\hline 07005000 & $\begin{array}{l}\text { Maline Creek at Bellfontaine Road in } \\
\text { Bellfontaine Place- }\end{array}$ & --- & $\ldots$ & --- & --- & --- & --- & --- & $x$ & --- & --- & $-\cdots-$ & ---- \\
\hline 07010016 & $\begin{array}{l}\text { River des Peres at Hafner Place in } \\
\text { University City- }\end{array}$ & $-\cdots$ & $-\cdots$ & --- & --- & --- & --- & --- & $x$ & $\cdots$ & $\cdots$ & $\cdots-$ & $\cdots$ \\
\hline 07010044 & Deer Creek at Warson Road in Ladue-- & --- & --- & --- & --- & --- & --- & --- & $x$ & --- & --- & --- & --- \\
\hline 07010086 & $\begin{array}{l}\text { Deer Creek at Big Bend Blvd. in } \\
\text { Maplewood- }\end{array}$ & $-\cdots$ & $-\cdots$ & $-\cdots$ & --- & --- & ---- & ---- & $x$ & --- & --- & --- & --- \\
\hline 07010155 & $\begin{array}{l}\text { Gravois Creek at Teshire Road at } \\
\text { Sappington- }\end{array}$ & --- & $\cdots$ & $-\cdots$ & --- & $-\cdots$ & --- & ---- & $x$ & --- & ---- & --- & --- \\
\hline 07011600 & Love Branch at Rolla- & --- & --- & $x$ & --- & $x$ & --- & --- & --- & --- & --- & --- & --- \\
\hline 07017115 & $\begin{array}{l}\text { Fox Creek at 01d Highway } 66 \text {, St. } \\
\text { Louis County-. }\end{array}$ & --- & --- & $-\cdots$ & $x$ & $x$ & --- & --- & --- & --- & --- & --- & --- \\
\hline 07019035 & $\begin{array}{l}\text { Forby Creek at Highway 109, St. } \\
\text { Louis County- }\end{array}$ & --- & --- & --- & $x$ & $x$ & --- & --- & --- & --- & --- & $-\cdots$ & --- \\
\hline 07019050 & $\begin{array}{l}\text { Hami } 1 \text { ton Creek at Highway 109, St. } \\
\text { Louis County-- }\end{array}$ & --- & --- & --- & $x$ & $x$ & --- & --- & --- & ---- & --- & --- & --- \\
\hline 07019070 & $\begin{array}{l}\text { Kiefer Creek at New Ballwin Road, } \\
\text { St. Louis County-- }\end{array}$ & --- & --- & ---- & $x$ & $x$ & --- & ---- & ---- & --- & $\cdots$ & $\cdots$ & $\cdots$ \\
\hline 07019100 & $\begin{array}{l}\text { Fishpot Creek at 01d Ballwin Road, } \\
\text { St. Louis County- }\end{array}$ & --- & ---- & ---- & $x$ & $x$ & --- & --- & --- & --- & --- & --- & --- \\
\hline 07019120 & $\begin{array}{l}\text { Fishpot Creek at Hanna Road, St. } \\
\text { Louis County-- }\end{array}$ & --- & --- & --- & $x$ & $x$ & --- & --- & --- & --- & --- & --- & --- \\
\hline 07019145 & $\begin{array}{l}\text { Grand G1aize Creek at Highway 141, St. } \\
\text { Louis County }\end{array}$ & --- & --- & --- & $x$ & $x$ & --- & --- & $-\cdots$ & --- & --- & --- & ---- \\
\hline 07019180 & $\begin{array}{l}\text { Grand G1aize Creek at Doughtery Ferry } \\
\text { Road, St. Louis County }\end{array}$ & --- & --- & --- & $x$ & $x$ & --- & --- & ---- & --- & --- & --- & --- \\
\hline 07019320 & $\begin{array}{l}\text { Mattese Creek at Yaeger Road, St. } \\
\text { Louis County-. }\end{array}$ & $-\cdots$ & --- & ---- & $x$ & $x$ & ---- & --- & --- & --- & --- & --- & --- \\
\hline
\end{tabular}


Table 3.--Urban rainfal1-runoff station network, 1979--continued

[DG\&LS, Division of Geology and Land Survey; City of Spfd., City of Springfield; Mo. Highway Comm., Missouri Highway Commission; St. Louis Co., St. Louis County; USGS Coop, U.S. Geological Survey Coop; USGS Fed., U.S. Geological Survey-Federal; CE RI, Corps of Engineers, Rock Island; CE STL, Corps of Engineers, St. Louis; CE KC, Corps of Engineers, Kansas City; CE TU, Corps of Engineers, Tulsa; CE LR, Corps of Engineers, Little Rock; UE, Union Electric]

\begin{tabular}{|c|c|c|c|c|c|c|c|c|c|c|c|c|c|}
\hline \multirow[b]{2}{*}{$\begin{array}{l}\text { Station } \\
\text { no. }\end{array}$} & \multirow[b]{2}{*}{ Station name } & \multicolumn{10}{|c|}{ SOURCE OF FUNDING } & \multicolumn{2}{|c|}{ 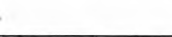 } \\
\hline & & DG\&LS & $\begin{array}{l}\text { City } \\
\text { of } \\
\text { Spfd. }\end{array}$ & $\begin{array}{l}\text { High- } \\
\text { way } \\
\text { Comm. }\end{array}$ & $\begin{array}{l}\text { St. } \\
\text { Louis } \\
\text { Co. }\end{array}$ & $\begin{array}{l}\text { USGS } \\
\text { Coop }\end{array}$ & $\begin{array}{l}\text { USGS } \\
\text { Fed. }\end{array}$ & CE RI & CE STL & CE KC & CE TU & CE LR & UE \\
\hline 07020860 & $\begin{array}{l}\text { Cape la Croix at Highway } 61 \text { in Cape } \\
\text { Girardeau- }\end{array}$ & --- & --- & --- & --- & --- & --- & --- & $x$ & $-\cdots$ & --- & ---- & --- \\
\hline 07020870 & $\begin{array}{l}\text { Cape la Croix at Bloomfield in } \\
\text { Cape Girardeau- }\end{array}$ & --- & --- & --- & --- & --- & --- & --- & $x$ & --- & --- & --- & --- \\
\hline 07052000 & $\begin{array}{l}\text { Wilsons Creek at Scenic Drive at } \\
\text { Springfield- }\end{array}$ & $-\cdots$ & $x$ & --- & --- & $x$ & $-\cdots$ & --- & --- & --- & --- & ---- & --- \\
\hline 07052050 & $\begin{array}{c}\text { North Fork Wilsons Creek at Highways } \\
13 \& 166 \text { at Springfield-- }\end{array}$ & --- & $x$ & --- & --- & $x$ & --- & --- & --- & --- & --- & --- & --- \\
\hline
\end{tabular}


Table 4.--Active low-flow partial-record stations, 1979

[DG\&LS, Division of Geology and Land Survey; City of Spfd., City of Springfield; Mo. Highway Comm., Missouri Highway Commission; Mo. Conserv. Comm., Missouri Conservation Commission; USGS Coop, U.S. Geological Survey Coop; USGS Fed., U.S. Geological Survey-Federal; CE RI, Corps of Engineers, Rock Island; CE STL, Corps of Engineers, St. Louis; CE KC, Corps of Engineers, Kansas City; CE TU, Corps of Engineers, Tulsa; CE LR, Corps of Engineers, Little Rock; UE, Union Electric]

\begin{tabular}{|c|c|c|c|c|c|c|c|c|c|c|c|c|c|}
\hline \multirow[b]{2}{*}{$\begin{array}{l}\text { Station } \\
\text { no. }\end{array}$} & \multirow[b]{2}{*}{ Station name } & \multicolumn{12}{|c|}{ SOURCE OF FUNDING } \\
\hline & & DG\&LS & $\begin{array}{l}\text { City } \\
\text { of } \\
\text { Spfd. }\end{array}$ & $\begin{array}{l}\text { Mo. } \\
\text { High- } \\
\text { way } \\
\text { Comm. }\end{array}$ & $\begin{array}{l}\text { Mo. } \\
\text { Conserv. } \\
\text { Comm. }\end{array}$ & $\begin{array}{l}\text { USGS } \\
\text { Coop }\end{array}$ & $\begin{array}{l}\text { USGS } \\
\text { Fed. }\end{array}$ & CE RI & CE STL & CE KC & CE TU & CE LR & UE \\
\hline 05497300 & North Fabius River near Durham------ & $x$ & --- & --- & --- & $x$ & --- & --- & --- & --- & --- & --- & --- \\
\hline 05498300 & Middle Fabius River near Durham----- & $x$ & --- & --- & --- & $x$ & --- & --- & --- & --- & --- & --- & --- \\
\hline 05498500 & North Fabius River at Taylor------ & $x$ & --- & --- & --- & $x$ & --- & --- & --- & --- & ---- & --- & --- \\
\hline 05509300 & Salt River near Ashburn-- & $x$ & --- & --- & --- & $x$ & $-\cdots$ & --- & --- & --- & $-\cdots$ & --- & --- \\
\hline 06810050 & High Creek near Rock Port-- & $x$ & --- & --- & --- & $x$ & --- & --- & --- & --- & --- & --- & --- \\
\hline 06811600 & Rock Creek near Rock Port-- & $x$ & --- & --- & --- & $x$ & --- & --- & --- & --- & --- & --- & --- \\
\hline 06815575 & Squaw Creek near Mound City------- & $x$ & --- & --- & ---- & $x$ & --- & --- & --- & --- & --- & --- & --- \\
\hline 06817700 & Nodaway River near Graham--_--- & $x$ & --- & --- & --- & $x$ & --- & --- & --- & --- & --- & --- & --- \\
\hline 06817800 & Nodaway River near Oregon--.-- & $x$ & --- & --- & --- & $x$ & --- & --- & --- & --- & ---- & --- & --- \\
\hline 06818490 & Bee Creek near Platte City-_- & $x$ & --- & --- & --- & $x$ & --- & --- & --- & $-\cdots$ & --- & --- & --- \\
\hline 06819090 & Platte River near St. Joseph-- & $x$ & --- & --- & --- & $x$ & --- & --- & --- & --- & --- & --- & --- \\
\hline 06820460 & $\begin{array}{l}\text { One Hundred and Two River at Avenue } \\
\text { City- }\end{array}$ & $x$ & --- & --- & --- & $x$ & --- & --- & --- & --- & --- & --- & --- \\
\hline 06820480 & $\begin{array}{l}\text { One Hundred and Two River near St. } \\
\text { Joseph- }\end{array}$ & $x$ & --- & ---- & --- & $x$ & ---- & $-\cdots$ & ---- & --- & $-\cdots$ & --- & --- \\
\hline 06820490 & Third Fork Platte River near Easton-- & $x$ & --- & --- & --- & $x$ & $-\cdots$ & ---- & ---- & ---- & --- & --- & --- \\
\hline 06821200 & Platte River at Platte City--- & $x$ & ---- & --- & --- & $x$ & --- & $-\cdots$ & --- & --- & --- & --- & --- \\
\hline 06893900 & Little Blue River near Blue Springs-- & $x$ & --- & --- & --- & $x$ & --- & --- & --- & --- & --- & --- & --- \\
\hline 06894800 & Sni-A-Bar Creek near Wellington--.--- & $x$ & --- & --- & --- & $x$ & --- & --- & --- & --- & --- & --- & --- \\
\hline 06895000 & Crooked River near Richmond-_- & $x$ & --- & --- & --- & $x$ & --- & --- & --- & --- & --- & $-\cdots$ & --- \\
\hline 06896182 & Wildcat Creek at Stanberry--.--- & $x$ & --- & --- & --- & $x$ & --- & --- & --- & --- & --- & --- & --- \\
\hline 06896550 & Grand River near Darlington----- & $x$ & --- & --- & --- & $x$ & --- & $-\cdots$ & --- & --- & --- & --- & --- \\
\hline 06896650 & Sampson Creek at Pattonsburg------ & $x$ & --- & --- & --- & $x$ & --- & --- & --- & --- & --- & --- & --- \\
\hline 06896900 & Grand River near Pattonsburg------ & $x$ & --- & ---- & --- & $x$ & --- & --- & --- & --- & --- & --- & --- \\
\hline
\end{tabular}


Table 4.--Active low-flow partial-record stations, 1979--continued

[DG\&LS, Division of Geology and Land Survey; City of Spfd., City of Springfield; Mo. Highway Comm., Missouri Highway Commission; Mo. Conserv. Comm., Missouri Conservation Commission; USGS Coop, U.S. Geological Survey Coop; USGS Fed., U.S. Geological Survey-Federal; CE RI, Corps of Engineers, Rock Island; CE STL, Corps of Engineers, St. Louis; CE KC, Corps of Engineers, Kansas City; CE TU, Corps of Engineers, Tulsa; CE LR, Corps of Engineers, Little Rock; UE, Union Electric]

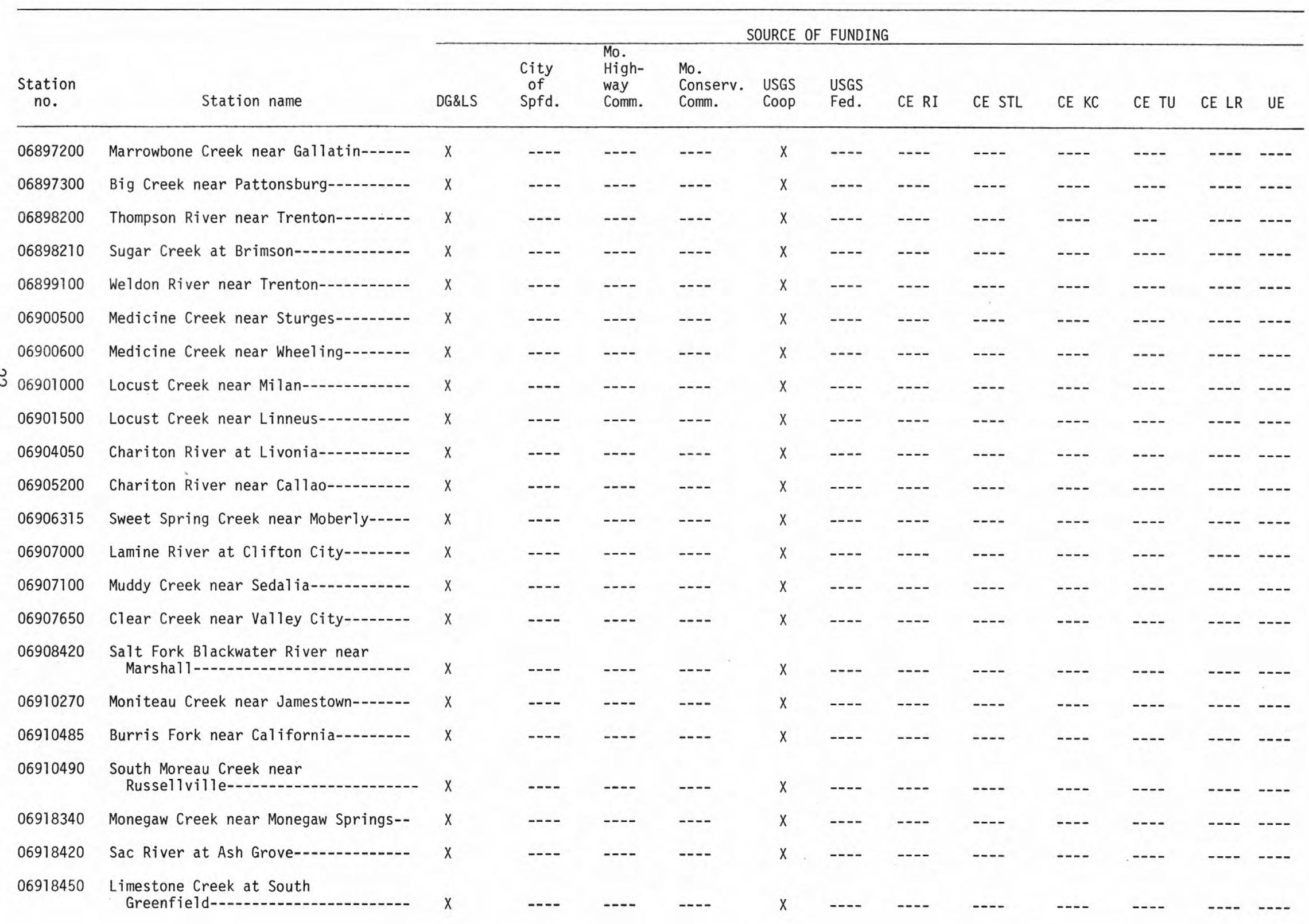


Table 4.--Active low-flow partial-record stations, 1979--continued

[DG\&LS, Division of Geology and Land Survey; City of Spfd., City of Springfield; Mo. Highway Comm., Missouri Highway Commission; Mo. Conserv. Comm., Missouri Conservation Commission; USGS Coop, U.S. Geological Survey Coop; USGS Fed., U.S. Geological Survey-Federa 1; CE RI, Corps of Engineers, Rock Island; CE STL, Corps of Engineers, St. Louis; CE KC, Corps of Engineers, Kansas City; CE TU, Corps of Engineers, Tulsa; CE LR, Corps of Engineers, Little Rock; UE, Union Electric]

\begin{tabular}{|c|c|c|c|c|c|c|c|c|c|c|c|c|c|}
\hline \multirow[b]{2}{*}{$\begin{array}{l}\text { Station } \\
\text { no. }\end{array}$} & \multirow[b]{2}{*}{ Station name } & \multicolumn{11}{|c|}{ SOURCE OF FUNDING } & \multirow[b]{2}{*}{ UE } \\
\hline & & DG\&LS & $\begin{array}{l}\text { City } \\
\text { of } \\
\text { Spfd. }\end{array}$ & $\begin{array}{l}\text { Mo. } \\
\text { High- } \\
\text { way } \\
\text { Comm. }\end{array}$ & $\begin{array}{l}\text { Mo. } \\
\text { Conserv. } \\
\text { Comm. }\end{array}$ & $\begin{array}{l}\text { USGS } \\
\text { Coop }\end{array}$ & $\begin{array}{l}\text { USGS } \\
\text { Fed. }\end{array}$ & CE RI & CE STL & CE KC & CE TU & CE LR & \\
\hline 06920600 & Weaubleau Creek near Osceola------- & $x$ & --- & --- & --- & $x$ & --- & --- & ---- & --- & --- & --- & ---- \\
\hline 06920850 & Hogles Creek near Fairfield-------- & $x$ & --- & --- & --- & $x$ & --- & --- & --- & --- & --- & --- & ---- \\
\hline 06921000 & Pomme de Terre River near Bolivar-- & $x$ & --- & ---- & ---- & $x$ & ---- & ---- & ---- & --- & ---- & ---- & ---- \\
\hline 06922580 & Big Turkey Creek near Warsaw------- & $x$ & --- & ---- & --- & $x$ & ---- & ---- & --- & ---- & ---- & --- & ---- \\
\hline 06922780 & Deer Creek near Edwards--.-.--- & $x$ & --- & --- & --- & $x$ & ---- & ---- & --- & ---- & $-\cdots$ & --- & --- \\
\hline 06923200 & Niangua River near Buffalo--------- & $x$ & --- & --- & --- & $x$ & --- & ---- & --- & --- & --- & --- & --- \\
\hline 06923250 & Niangua River near Windyville------- & $x$ & --- & ---- & ---- & $x$ & ---- & ---- & ---- & $-\cdots$ & ---- & --- & ---- \\
\hline U.jo6923900 & Niangua River near Eldridge---.----- & $x$ & --- & ---- & ---- & $x$ & ---- & --- & ---- & --- & $\cdots$ & ---- & ---- \\
\hline 06925250 & $\begin{array}{l}\text { Little Niangua River near Macks } \\
\text { Creek- }\end{array}$ & $x$ & --- & --- & --- & $x$ & --- & --- & --- & $-\cdots$ & $-\cdots$ & --- & --- \\
\hline 06925440 & Grandglaize Creek near Brumley----- & $x$ & --- & ---- & ---- & $x$ & ---- & ---- & ---- & ---- & ---- & ---- & ---- \\
\hline 06926160 & Saline Creek near Tuscumbia-------- & $x$ & ---- & ---- & ---- & $x$ & ---- & ---- & ---- & --- & --- & ---- & ---- \\
\hline 06926250 & $\begin{array}{l}\text { Little Tavern Creek near St. } \\
\text { Elizabeth- }\end{array}$ & $x$ &.-- & --- & ---- & $x$ & --- & --- & --- & --- & --- & --- & ---- \\
\hline 06926700 & Maries River near Vienna---.------ & $x$ & ---- & --- & ---- & $x$ & ---- & --- & --- & ---- & ---- & --- & --- \\
\hline 06926820 & Little Maries River near Vienna---- & $x$ & --- & ---- & ---- & $x$ & ---- & ---- & $-\cdots$ & --- & --- & --- & ---- \\
\hline 06927000 & Maries River at Westphalia-...-..-- & $x$ & --- & ---- & ---- & $x$ & --- & --- & ---- & --- & --- & --- & ---- \\
\hline 06927520 & Bailey Creek at Morrison----------- & $x$ & ---- & ---- & ---- & $x$ & ---- & ---- & --- & ---- & ---- & --- & ---- \\
\hline 06927580 & Gasconade River near Hartville----- & $x$ & ---- & ---- & ---- & $x$ & ---- & ---- & ---- & --- & --- & ---- & ---- \\
\hline 06927650 & Beaver Creek near Manes---------- & $x$ & ---- & ---- & ---- & $x$ & --- & ---- & ---- & --- & ---- & ---- & ---- \\
\hline 06927730 & 0sage Fork at Rader-- & $x$ & --- & --- & --- & $x$ & --- & --- & --- & $-\cdots$ & --- & --- & ---- \\
\hline 06927750 & Osage Fork near Orla-...- & $x$ & --- & --- & --- & $x$ & --- & --- & --- & --- & --- & --- & --- \\
\hline 06928000 & Gasconade River near Hazelgreen---- & $x$ & $-\cdots$ & --- & --- & $x$ & --- & --- & --- & --- & --- & --- & --- \\
\hline 06928300 & $\begin{array}{l}\text { Roubidoux Creek at Fort Leonard } \\
\text { Wood- }\end{array}$ & $x$ & --- & --- & --- & $x$ &.-- & --- & --- & --- & --- & --- & $\cdots$ \\
\hline
\end{tabular}


Table 4.--Active low-flow partial-record stations, 1979--continued

[DG\&LS, Division of Geology and Land Survey; City of Spfd., City of Springfield; Mo. Highway Comm., Missouri Highway Commission; Mo. Conserv. Comm., Missouri Conservation Commission; USGS Coop, U.S. Geological Survey Coop; USGS Fed., U.S. Geological Survey-Federal; CE RI, Corps of Engineers, Rock Island; CE STL, Corps of Engineers, St. Louis; CE KC, Corps of Engineers, Kansas City; CE TU, Corps of Engineers, Tulsa; CE LR, Corps of Engineers, Little Rock; UE, Union Electric]

\begin{tabular}{|c|c|c|c|c|c|c|c|c|c|c|c|c|c|}
\hline \multirow[b]{2}{*}{$\begin{array}{l}\text { Station } \\
\text { no. }\end{array}$} & \multirow[b]{2}{*}{ Station name } & \multicolumn{12}{|c|}{ SOURCE OF FUNDING } \\
\hline & & DG\&LS & $\begin{array}{l}\text { City } \\
\text { of } \\
\text { Spfd. }\end{array}$ & $\begin{array}{l}\text { Migh- } \\
\text { way } \\
\text { Comm. }\end{array}$ & $\begin{array}{l}\text { Mo. } \\
\text { Conserv. } \\
\text { Comm. }\end{array}$ & $\begin{array}{l}\text { USGS } \\
\text { Coop }\end{array}$ & $\begin{array}{l}\text { USGS } \\
\text { Fed. }\end{array}$ & CE RI & CE STL & CE KC & CE TU & CE LR & UE \\
\hline 06928500 & Gasconade River near Waynesville----- & $x$ & $-\cdots$ & $-\cdots$ & $-\cdot--$ & $x$ & $-\cdots$ & $-\cdots$ & $-\cdots$ & --- & $-\cdots$ & --- & --- \\
\hline 06929300 & Big Piney River near Licking---------- & $x$ & $-\cdots$ & ---- & --- & $x$ & ---- & --- & --- & --- & --- & ---- & ---- \\
\hline 06933790 & 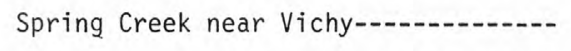 & $x$ & ---- & ---- & --- & $x$ & ---- & ---- &.-- & --- & --- & --- & --- \\
\hline 06934700 & Loutre River at McKittrick---------- & $x$ & ---- & ---- & ---- & $x$ & ---- & --- & --- & --- & --- & ---- & --- \\
\hline 06935300 & Charette Creek near Marthasville----- & $x$ & ---- & ---- & --- & $x$ & ---- & ---- & ---- & ---- & --- &.-- & --- \\
\hline 07010120 & Gravois Creek near Kirkwood---------- & $x$ & --- & --- & $-\cdots$ & $x$ & ---- & ---- & --- & $-\cdots$ & ---- & ---- & ---- \\
\hline 07014100 & Courtois Creek at Courtois---.----- & $x$ & ---- & ---- & ---- & $x$ & ---- & ---- & ---- & ---- & --- & --- & --- \\
\hline 07014130 & Indian Creek at Courtois----.---- & $x$ & --- & ---- & ---- & $x$ & ---- & ---- & --- & --- & --- & ---- & --- \\
\hline 07014800 & Indian Creek near St. Clair-......-- & $x$ & --- & --- & $-\cdots$ & $x$ & --- & --- & --- & --- & ---- & ---- & ---- \\
\hline 07015780 & $\begin{array}{l}\text { Little Bourbeuse River near } \\
\text { Sullivan-....- }\end{array}$ & $x$ & $-\cdots$ & --- & $-\cdots$ & $x$ & $-\cdots$ & --- & --- & --- & --- & $-\cdots$ & --- \\
\hline 07017650 & Fourche-A-Renault Creek near Potosi-- & $x$ & --- & --- & --- & $x$ & ---- & ---- & ---- & --- & ---- & --- & ---- \\
\hline 07017800 & Mineral Fork near Potosi---------.-- & $x$ & ---- & --- & ---- & $x$ & --- & --- & $-\cdots$ & --- & ---- & ---- & ---- \\
\hline 07017900 & 01d Mines Creek near Potosi---------- & $x$ & --- & --- & --- & $x$ & --- & --- & ---- & --- & --- & --- & ---- \\
\hline 07020100 & Establishment Creek at Bloomsdale---- & $x$ & --- & --- & $-\cdots$ & $x$ & $-\cdots$ & --- & --- & --- & ---- & --- & -.-- \\
\hline 07020250 & River AuxVases near St. Genevieve---- & $x$ & ---- & --- & $-\cdots$ & $x$ & --- & $-\cdots$ & --- & $-\cdots$ & --- & --- & --- \\
\hline 07020270 & Saline Creek near Minnith---.----- & $x$ & --- & --- & --- & $x$ & --- & ---- & --- & --- & ---- & ---- & ---- \\
\hline 07020300 & Saline Creek near St. Marys-----.-- & $x$ & --- & $-\cdots$ & --- & $x$ & ---- & --- & $-\cdots$ & $-\cdots$ & $-\cdots$ & $-\cdots$ & --- \\
\hline 07020600 & Apple Creek at Appleton--..--..-- & $x$ & --- & $-\cdots$ & $-\cdots$ & $x$ & ---- & ---- & ---- & --- & ---- & ---- & ---- \\
\hline 07020750 & Indian Creek near Fruitland---------- & $x$ & --- & --- & $-\cdots$ & $x$ & $-\cdots$ & $\cdots$ & --- & --- & --- & $-\cdots$ & --- \\
\hline 07020950 & Castor River near Cascade---.------ & $x$ & ---- & ---- & --- & $x$ & $-\cdots$ & --- & ---- & --- & --- & --- & --- \\
\hline 07020970 & Bear Creek near Lowndes--------- & $x$ & --- & --- & --- & $x$ & --- & --- & --- & $-\cdots$ & --- & ---- & ---- \\
\hline 07021500 & $\begin{array}{l}\text { Little Whitewater River near } \\
\text { Millersville-- }\end{array}$ & $x$ & --- & --- & --- & $x$ & ---- & --- & ---- & --- & --- & --- & ---- \\
\hline
\end{tabular}


Table 4.--Active low-flow partial-record stations, 1979--continued

[DG\&LS, Division of Geology and Land Survey; City of Spfd., City of Springfield; Mo. Highway Comm., Missouri Highway

Commission; Mo. Conserv. Comm., Missouri Conservation Commission; USGS Coop, U.S. Geological Survey Coop; USGS Fed., U.S. Geological Survey-Federal; CE RI, Corps of Engineers, Rock Island; CE STL, Corps of Engineers, St. Louis; CE KC, Corps of Engineers, Kansas City; CE TU, Corps of Engineers, Tulsa; CE LR, Corps of Engineers, Little Rock; UE, Union Electric]

\begin{tabular}{|c|c|c|c|c|c|c|c|c|c|c|c|c|c|}
\hline \multirow[b]{2}{*}{$\begin{array}{l}\text { Station } \\
\text { no. }\end{array}$} & \multirow[b]{2}{*}{ Station name } & \multicolumn{12}{|c|}{ SOURCE OF FUNDING } \\
\hline & & DG\&LS & $\begin{array}{l}\text { City } \\
\text { of } \\
\text { Spfd. }\end{array}$ & $\begin{array}{l}\text { High- } \\
\text { way } \\
\text { Comm. }\end{array}$ & $\begin{array}{l}\text { Mo. } \\
\text { Conserv. } \\
\text { Comm. }\end{array}$ & $\begin{array}{l}\text { USGS } \\
\text { Coop }\end{array}$ & $\begin{array}{l}\text { USGS } \\
\text { Fed. }\end{array}$ & CE RI & CE STL & CE KC & CE TU & CE LR & UE \\
\hline 07021530 & 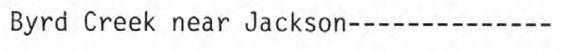 & $x$ & --- & --- & --- & $x$ & --- & --- & --- & --- & --- & --- & --- \\
\hline 07021800 & $\begin{array}{l}\text { Headwater diversion channel near } \\
\text { Allenville- }\end{array}$ & $x$ & $-\cdots$ & --- & --- & $x$ & --- & --- & --- & $-\cdots$ & --- & --- & --- \\
\hline 07024100 & Wilkerson ditch near East Prairie---- & $x$ & --- & --- & --- & $x$ & --- & --- & --- & --- & --- & --- & --- \\
\hline 07024150 & St. James ditch at East Prairie------ & $x$ & --- & --- & --- & $x$ & --- & --- & --- & --- & --- & --- & --- \\
\hline 07036090 & Twelvemile Creek near Annapolis------ & $x$ & --- & --- & --- & $x$ & $-\ldots$ & $-\cdots$ & --- & $-\ldots$ & $-\ldots$ & --- & --- \\
\hline 07036100 & St. Francis River near Annapolis----- & $x$ & --- & --- & --- & $x$ & --- & --- & --- & --- & --- & --- & $-\cdots$ \\
\hline 07037200 & Crane Pond Creek near Annapolis-...- & $x$ & --- & --- & --- & $x$ & --- & --- & $-\cdots$ & --- & --- & --- & --- \\
\hline 07040700 & Ditch 9 near Gideon- & $x$ & $-\cdots$ & $-\cdots$ & --- & $x$ & --- & --- & --- & --- & --- & --- & --- \\
\hline 07040800 & Main ditch 6 east of Malden-1.-- & $x$ & --- & --- & --- & $x$ & --- & --- & --- & --- & --- & --- & --- \\
\hline 07040850 & Main ditch near Bernie-- & $x$ & --- & --- & --- & $x$ & --- & --- & --- & --- & --- & --- & --- \\
\hline 07040900 & Main ditch 2 at Malden & $x$ & --- & --- & --- & $x$ & --- & --- & --- & --- & --- & --- & --- \\
\hline 07041050 & Main ditch near Malden & $x$ & ---- & --- & --- & $x$ & ---- & ---- & --- & --- & ---- & --- & --- \\
\hline 07041100 & 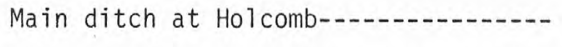 & $x$ & --- & ---- & ---- & $x$ & --- & ---- & --- & $-\cdots$ & --- & --- & --- \\
\hline 07042400 & Main ditch 1 near Matthews-........... & $x$ & $-\cdots$ & --- & --- & $x$ & --- & --- & --- & --- & --- & --- & --- \\
\hline 07043050 & Ditch 24 at Heagy- & $x$ & --- & --- & --- & $x$ & --- & --- & --- & --- & --- & --- & --- \\
\hline 07043100 & 01d Channel ditch 1 near Chaffee----- & $x$ & --- & --- & --- & $x$ & --- & --- & --- & --- & --- & --- & --- \\
\hline 07046510 & Pemiscot Bayou near Holland-...-- & $x$ & --- & --- & --- & $x$ & --- & --- & --- & --- & --- & --- & --- \\
\hline 07046520 & Main ditch 1 near Deering-_.......- & $x$ & --- & --- & --- & $x$ & --- & --- & $-\cdots$ & $-\cdots$ & $-\cdots$ & --- & --- \\
\hline 07050540 & James River near Northview-.......- & $x$ & --- & --- & --- & $x$ & --- & --- & --- & --- & --- & --- & --- \\
\hline 07050560 & Panther Creek near Northview-..-.-- & $x$ & --- & --- & --- & $x$ & $-\cdots$ & $-\cdots$ & --- & --- & $-\cdots$ & --- & --- \\
\hline 07052260 & Finley Creek near Linden-1...- & $x$ & --- & --- & --- & $x$ & --- & --- & --- & --- & --- & --- & --- \\
\hline 07052360 & Crane Creek near Galena--...-..-- & $x$ & --- & --- & --- & $x$ & --- & --- & --- & --- & --- & --- & --- \\
\hline 07052900 & Flat Creek near Cape Fair--- & $x$ & --- & --- & --- & $x$ & --- & --- & --- & --- & --- & --- & ---- \\
\hline
\end{tabular}


[DG\&LS, Division of Geology and Land Survey; City of Spfd., City of Springfield; Mo. Highway Comm., Missouri Highway Commission; Mo. Conserv. Comm., Missouri Conservation Commission; USGS Coop, U.S. Geological Survey Coop; USGS Fed., U.S. Geological

Survey-Federal; CE RI, Corps of Engineers, Rock Island; CE STL, Corps of Engineers, St. Louis; CE KC, Corps of Engineers, Kansas City; CE TU, Corps of Engineers, Tulsa; CE LR, Corps of Engineers, Little Rock; UE, Union Electric]

\begin{tabular}{|c|c|c|c|c|c|c|c|c|c|c|c|c|c|}
\hline \multirow[b]{2}{*}{$\begin{array}{l}\text { Station } \\
\text { no. }\end{array}$} & \multirow[b]{2}{*}{ Station name } & \multicolumn{11}{|c|}{ SOURCE OF FUNDING } & \multirow[b]{2}{*}{ UE } \\
\hline & & DG\&LS & $\begin{array}{l}\text { City } \\
\text { of } \\
\text { Spfd. }\end{array}$ & $\begin{array}{l}\text { High- } \\
\text { way } \\
\text { Comm. }\end{array}$ & $\begin{array}{l}\text { Mo. } \\
\text { Conserv. } \\
\text { Comm. }\end{array}$ & $\begin{array}{l}\text { USGS } \\
\text { Coop }\end{array}$ & $\begin{array}{l}\text { USGS } \\
\text { Fed. }\end{array}$ & CE RI & CE STL & CE KC & CE TU & CE LR & \\
\hline 07053850 & Swan Creek at Garrison-- & $x$ & --- & --- & --- & $x$ & --- & --- & --- & --- & --- & --- & $\overline{---}$ \\
\hline 07054020 & Beaver Creek near Ava-..- & $x$ & ---- & --- & --- & $x$ & --- & --- & --- & --- & --- & --- & --- \\
\hline 07054040 & Beaver Creek near Bradleyville------ & $x$ & --- & --- & --- & $x$ & --- & --- & --- & --- & --- & --- & --- \\
\hline 07057470 & Nöth Fork River near Dora--.--- & $x$ & --- & --- & --- & $x$ & $-\cdots$ & --- & --- & --- & --- & --- & --- \\
\hline 07057480 & Spring Creek near Sycamore-.-...- & $x$ & --- & --- & ---- & $x$ & --- & $-\cdots$ & --- & --- & --- & $-\cdots$ & --- \\
\hline 07057680 & Hunter Creek at Vera Cruz--.-- & $x$ & --- & --- & --- & $x$ & --- & --- & --- & --- & --- & --- & --- \\
\hline 07057700 & Bryant Creek near Evans-------- & $x$ & --- & --- & --- & $x$ & --- & --- & --- & --- & --- & --- & --- \\
\hline 07063100 & Lake Slough near Qulin & $x$ & $-\cdots$ & $-\cdots$ & --- & $x$ & --- & --- & --- & --- & --- & --- & --- \\
\hline 07063130 & Menorkenut Slough near Qulin------ & $x$ & --- & --- & --- & $x$ & --- & --- & --- & --- & --- & --- & --- \\
\hline 07063500 & Cane Creek at Harviell-- & $x$ & --- & --- & --- & $x$ & --- & --- & --- & --- & --- & $-\cdots$ & --- \\
\hline 07064480 & $\begin{array}{l}\text { Ashley Creek near Montauk State } \\
\text { Park- }\end{array}$ & $x$ & --- & --- & --- & $x$ & --- & --- & $-\cdots$ & --- & --- & --- & --- \\
\hline 07064520 & Big Creek at Cedar Grove---.-- & $x$ & --- & --- & --- & $x$ & --- & --- & --- & --- & --- & --- & --- \\
\hline 07064540 & Gladden Creek at Akers & $x$ & --- & --- & --- & $x$ & --- & --- & --- & --- & --- & --- & $-\cdots$ \\
\hline 07064750 & Sinking Creek near Shannondale--.-- & $x$ & --- & --- & --- & $x$ & --- & --- & --- & $-\cdots$ & --- & --- & --- \\
\hline 07064770 & Barren Creek near Shannondale------- & $x$ & --- & --- & --- & $x$ & --- & --- & --- & --- & --- & --- & --- \\
\hline 07065050 & Big Creek near Round Spring------- & $x$ & --- & --- & --- & $x$ & --- & --- & --- & --- & --- & --- & --- \\
\hline 07065950 & Mahans Creek at West Eminence------- & $x$ & --- & --- & --- & $x$ & --- & --- & --- & --- & --- & --- & --- \\
\hline 07066100 & Shawnee Creek near Eminence--..-.-- & $x$ & --- & --- & --- & $x$ & --- & --- & --- & --- & --- & $-\cdots$ & --- \\
\hline 07066520 & Blair Creek near Round Spring------ & $x$ & --- & --- & --- & $x$ & --- & --- & --- & --- & $-\cdots$ & --- & --- \\
\hline 07066600 & Rocky Creek near Eminence-------- & $x$ & --- & $-\cdots$ & --- & $x$ & --- & --- & --- & --- & --- & --- & --- \\
\hline 07066750 & Pine Valley Creek near Van Buren---- & $x$ & --- & --- & --- & $x$ & --- & --- & --- & --- & --- & --- & --- \\
\hline 07066990 & Pike Creek at Van Buren-1- & $x$ & --- & --- & --- & $x$ & --- & --- & --- & --- & --- & $-\cdots$ & $\ldots$ \\
\hline 07068500 & $\begin{array}{l}\text { Little Black River near } \\
\text { Fairdealing- }\end{array}$ & $x$ & --- & --- & --- & $x$ & --- & --- & --- & --- & --- & --- & --- \\
\hline
\end{tabular}




\section{Table 4.--Active low-flow partial-record stations, 1979--continued}

[DG\&LS, Division of Geology and Land Survey; City of Spfd., City of Springfield; Mo. Highway Comm., Missouri Highway Commission; Mo. Conserv. Comm., Missouri Conservation Commission; USGS Coop, U.S. Geological Survey Coop; USGS Fed., U.S. Geological Survey-Federal; CE RI, Corps of Engineers, Rock Island; CE STL, Corps of Engineers, St. Louis; CE KC, Corps of Engineers, Kansas City; CE TU, Corps of Engineers, Tulsa; CE LR, Corps of Engineers, Little Rock; UE, Union Electric]

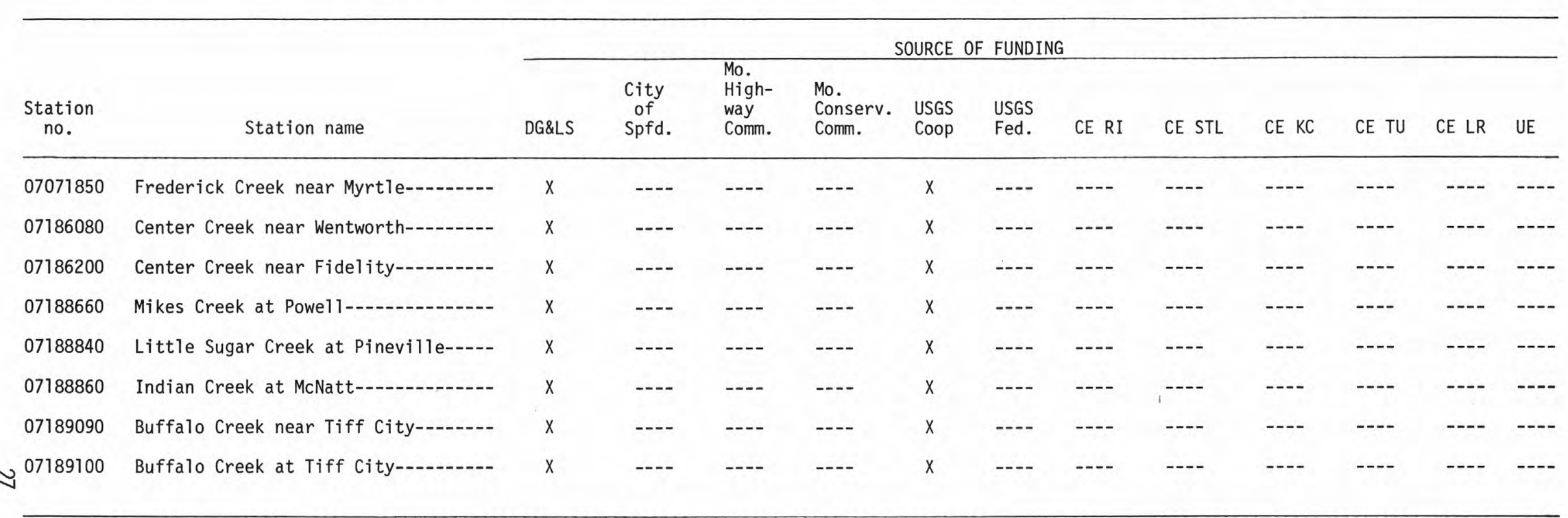


OBJECTIVES: To collect surface-water data sufficient to satisfy needs for current-purpose uses, such as (1) assessment of water resources, (2) operation of reservoirs or industries, (3) forecasting, (4) disposal of wastes and pollution controls, (5) discharge data to accompany water-quality measurements, (6) compact and legal requirements, and (7) research or special studies. To collect data necessary for analytical studies to define for any location the statistical properties of, and trends in, streamflow for use in planning and design.

APPROACH: $\quad$ Standard methods of data collection will be used as described in the series "Techniques of Water-Resources Investigations of the U.S. Geological Survey." Partialrecord gaging will be used instead of complete-record gaging where it serves the required purpose.

PROGRESS AND Streamflow data were collected as scheduled. SIGNIFICANT RESULTS:

PLANS FOR NEXT YEAR:

Collection of records to continue.

REPORTS : U.S. Geological Survey, 1978, Water-resources data for Missouri, water year 1977: U.S. Geological Survey Water-Data Report M0 77-1, 277 p. [Published annual1y.]

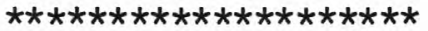

Professional Paper: Flood of September 1977 in Kansas City, Missouri and Kansas (Mø 00-001)

A Water-Resources Investigations report about the flood has been published (WRI 78-63). However, a report containing additional interpretive data is to be published as a Professional Paper. The Professional Paper has been prepared in cooperation with the Department of Commerce, National Weather Service, National Oceanic and Atmospheric Administration (NOAA) and is in review.
} 
Table 5.--Water-quality station network, 1979--continued

\begin{tabular}{|c|c|c|c|c|c|c|c|c|c|c|c|c|}
\hline \multirow[b]{2}{*}{$\begin{array}{l}\text { Station } \\
\text { no. }\end{array}$} & \multirow[b]{2}{*}{ Station name } & \multicolumn{11}{|c|}{ SOURCE OF FUNDING } \\
\hline & & $\begin{array}{l}\text { Type } \\
\text { of } \\
\text { record }\end{array}$ & DEQ & $\begin{array}{l}\text { Mo. } \\
\text { Conserv. } \\
\text { Comm. }\end{array}$ & $\begin{array}{l}\text { City } \\
\text { of } \\
\text { Spfd. }\end{array}$ & USGS & NPS & CE KC & CE STL & CE RI & CE TU & CE LR \\
\hline 07052100 & Wilsons Creek near Springfield--.--- & CT & -- & $-\cdots$ & $x$ & $x$ & -- & $-\cdots$ & $-\cdots--$ & $-\cdots$ & ----- & $\cdots-\cdots$ \\
\hline 07052160 & Wilsons Creek near Battlefield--.-- & $\mathrm{CT}$ & --- & $-\cdots$ & $x$ & $x$ & $-\cdots$ & $-\cdots$ & 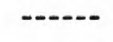 & $-\cdots$ & 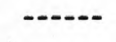 & $\cdots-\cdots$ \\
\hline 07052250 & James River near Boaz- & $\mathrm{CT}$ & $x$ & $-\cdots$ & $x$ & $x$ & -- & $-\cdots$ & $-\cdots$ & $-\cdots$ & -...- & $-\cdots$ \\
\hline 07053450 & $\begin{array}{l}\text { White River below Table Rock Dam } \\
\text { near Branson- }\end{array}$ & $\mathrm{CT}$ & --- & $\cdots$ & $-\cdots$ & $-\cdots$ & --- & $\cdots$ & $-\cdots--$ & $-\cdots-$ & -...- & $x$ \\
\hline 07053600 & Lake Taneycomo at School of the Ozarks-- & $\mathrm{CT}$ & --- & $-\cdots$ & --- & $-\cdots$ & -- & $-\cdots-$ & $-\cdots-$ & $-\cdots-$ & $-\cdots-$ & $x$ \\
\hline 07053700 & Lake Taneycomo at Branson & C & $x$ & $-\cdots$ & --- & $x$ & --- & $-\cdots$ & $-\ldots-n$ & $-\cdots-$ & $-\cdots$ & $-\cdots-$ \\
\hline 07053720 & Lake Taneycomo below Branson--..-- & CT & --- & ---- & --- & $-\cdots$ & --- & ----- & $-\cdots-$ & ---- &.--- & $x$ \\
\hline 07053825 & Lake Taneycomo at Forsyth & $\mathrm{CT}$ &.-- & $-\ldots$ & $-\cdots$ & -...- & $-\cdots$ & $-\ldots$ & $-\ldots$ & $-\ldots$ & $-\ldots$ & $x$ \\
\hline 07057500 & North Fork River near Tecumseh- & C & $x$ & $-\cdots$ & $-\cdots$ & $x$ & -- & $-\cdots$ & $-\cdots$ & $-\cdots$ & 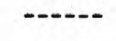 & $-\cdots$ \\
\hline 07064400 & Montauk Springs at Montauk & C & -- & $-\cdots$ & --- & $-\cdots$ & $x$ & $-\cdots$ & $-\cdots$ & $-\cdots$ & $-\cdots$ & \\
\hline 07064440 & Current River below Montauk State Pk---- & C & --- & $-\cdots$ & --- & ---- & $x$ & $-\cdots$ & $-\cdots$ & $-\cdots$ & $-\cdots$ & $-\ldots$ \\
\hline 07064530 & Welch Spring near Akers- & C & --- & $-\cdots$ & --- & ---- & $x$ & $-\cdots-$ & $-\cdots$ & $-\cdots$ & $-\cdots$ & $\cdots$ \\
\hline 07064555 & Pulltite Spring near Round Spring------ & $\mathrm{C}$ & -- & $-\cdots$ & --- & $\cdots$ & $x$ & $-\cdots$ & $-\cdots-1$ & $-\cdots$ & $\ldots$ & $\cdots$ \\
\hline 07065000 & Round Spring at Round Spring & C & --- & $-\cdots$ & --- & $-\cdots$ & $x$ & $-\cdots--$ & $-\cdots-1$ & $-\cdots-$ & $-\cdots--$ & $\cdots-\cdots$ \\
\hline 07065500 & Alley Spring at All ey- & C & --- & $-\cdots-$ & --- & ---- & $x$ & $-\cdots-$ & $-\cdots--$ & $-\cdots--$ & ---.- & $-\cdots-$ \\
\hline 07066110 & Jacks Fork above Two Rivers- & C & -- & $-\cdots$ & --- & $-\cdots$ & $x$ & $-\cdots--$ & $-\cdots--$ & $-\cdots--$ & ---- & $\cdots-\cdots$ \\
\hline 07066510 & Current River above Powder Mill------- & C & --- & $-\cdots$ & --- & $\cdots-$ & $x$ & $-\cdots$ & $-\cdots-$ & $-\cdots$ & $\cdots$ & 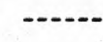 \\
\hline 07066550 & Blue Spring near Eminence- & C & --- & --- & --- & ---- & $x$ & $-\cdots$ & ---- & $--\cdots$ & $-\cdots--$ & $-\cdots--$ \\
\hline 07067500 & Big Spring near Van Buren--.-- & C & $-\cdots$ & ---- & --- & $-\cdots$ & $x$ & $--\cdot--$ & $-\ldots$ & $-\cdots$ & $-\cdots$ & $-\cdots-$ \\
\hline 07067800 & Current River below Hawes Campground---- & C & $\cdots$ & $\cdots$ & --- & $\cdots$ & $x$ & $\cdots$ & $-\cdots-\cdots$ & -....- & 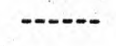 & $\cdots-\cdots$ \\
\hline 07068863 & Fourche River near Poynor- & $T$ & --- & $x$ & $-\cdots$ & $x$ & --- & ---- & $-\cdots--$ & $-\cdots--$ & $-\cdots-$ & $\ldots$ \\
\hline 07186250 & Grove Creek near Scotland & C & $-\cdots$ & $-\cdots$ & --- & $\cdots--$ & -- & $-\cdots$ & ---- & $-\cdots--$ & $x$ & $-\cdots-$ \\
\hline 07186400 & Center Creek near Carterville-------- & C & --- & $\cdots$ & $-\cdots$ & $\cdots--$ & --- & $-\cdots-$ & $-\cdots$ & ----- & $x$ & $\cdots-\cdots$ \\
\hline 07186480 & Center Creek near Smithfield-...-...- & $\mathrm{C}$ & $x$ & $-\ldots$ & --- & $x$ & -- & $-\cdots--$ & $-\ldots-$. & -...- & $-\ldots--$ & $-\cdots$ \\
\hline
\end{tabular}



PROJECT TITLE: Collection of ground-water data Mø 00-002

COOPERATOR: Department of Natural Resources, Division of Geology and Land Survey

LOCATION: $\quad$ Statewide

PROJECT CHIEF: L. F. Emmett

PROBLEM: $\quad$ Long-term water-level records are needed to evaluate the effects of climatic variations on the recharge to and discharge from the ground-water systems, to provide a data base from which to measure the effects of development, to assist in the prediction of future supplies, and to provide data for management of the resource.

OBJECTIVE: To collect water-level data sufficient to provide a minimum long-term data base so that the general response of the hydrologic system to natural climatic variations and induced stresses is known and potential problems can be defined early enough to allow proper planning and management. To provide a data base against which the short-term records acquired in areal studies can be analyzed. This analysis must (1) provide an assessment of the ground-water resource, (2) allow prediction of future conditions, (3) detect and define pollution and supply problems, and (4) provide the data base necessary for management of the resource.

APPROACH: $\quad$ Evaluation of regional geology allows broad, general definition of aquifer systems and their boundary conditions. Within this framework and with some knowledge of the stress on the system in time and space and the hydrologic properties of the aquifers, a subjective decision can be made on the most advantageous locations for observation of long-term system behavior. This subjective network can be refined as records become available and detailed areal studies of the ground-water system more closely define the aquifers, their properties, and the stresses to which they are subjected. 
PROGRESS AND SIGNIFICANT RESULTS:

PLANS FOR NEXT YEAR:
Many ground-water site schedules were converted from old Electronic Data Processing (EDP) system to System 2000. A network of about 100 alluvial wells in the Southeast Lowland were added to the observation well system for semi-annual measurement.

Continue converting we17-log records statewide to System 2000. Add observation points near the Jopl in mining district for semi-annual measurement.

PROJECT TITLE: Collection of quality-of-water data MØ 00-003

COOPERATORS: Department of Natural Resources

Division of Environmental Quality

Corps of Engineers

National Park Service

City of Springfield

LOCATION: $\quad$ Statewide

PROJECT CHIEF: J. H. Barks

PROBLEM: $\quad$ Water-resource planning and water-quality assessment require a statewide and nationwide base of relatively standardized information. For intelligent planning and realistic assessment of the water resource, the chemical and physical quality of the rivers and streams must be defined and monitored.

OBJECTIVE: $\quad$ To provide water-quality data for planning and action programs and to provide data for State and Federal management of interstate and international waters.

APPROACH: $\quad$ Operation of a network of water-quality stations to measure chemical concentrations, loads, and time trends as required by planning and management agencies.

PROGRESS AND SIGNIFICANT RESULTS: 
for the Corps of Engineers, and three monitors were operated in cooperation with the city of Springfield. A11 data collection continued on schedule.

PLANS FOR

NEXT YEAR:

REPORTS:

The water-quality network will continue approximately the same as the previous year. Several stations to measure daily suspended sediment will be added.

U.S. Geological Survey, 1978, Water-resources data for Missouri, water year 1977: U.S. Geological Survey Water-Data Report M0 77-1, 277 p. [Published annual1y.]

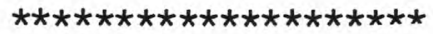

PROJECT TITLE: Flood Insurance Studies for Federal Insurance Administration, HUD MD 72-006

COOPERATOR: U.S. Department of Housing and Urban Development, Federal Insurance Administration.
\end{abstract}

LOCATION: $\quad$ Statewide

PROJECT CHIEF: L. D. Hauth

PROBLEM:

The National Flood Insurance Act of 1968 provides that the Department of Housing and Urban Development operate a flood insurance program through the Federal Insurance Administration. The Department of Housing and Urban Development (HUD) needs flood studies in selected areas to determine applicable flood insurance premium rates.

OBJECTIVE: To conduct the necessary hydrologic and hydraulic studies of the areas assigned by the Federal Insurance Administration (FIA), and to develop the most efficient procedures to attain the accuracy specified by FIA in the most appropriate format with minimum personnel requirements.

APPROACH: $\quad$ Conduct necessary surveys by ground and photogrammetric methods, prepare computer models of drainage networks, compute magnitudes and profiles of floods of specified frequencies, and furnish results in reports prepared to FIA specifications.

PROGRESS AND SIGNIFICANT

Type-15 studies for Salem and Willow Springs, Mo., are RESULTS: complete. Type-15 studies for Mounta in View and Ellington, Mo., have been submitted to HUD for preliminary publication. A study for Farmington, Mo., is nearly complete. 
PLANS FOR

NEXT YEAR:

REPORTS:

Manpower constraints make it unlikely that the project will continue beyond 1979 .

Published: Flood Insurance Study for Willows Springs, Missouri.

Flood Insurance Study for Salem, Missouri.

In Process: Flood Insurance Study for Ellington, Missouri.

Flood Insurance Study for Mountain View, Missouri.

Planned: Flood Insurance Study for Farmington, Missouri.

PROJECT TITLE: Planning a water-use data base system for Missouri Mø 79-007

COOPERATOR: Department of Natural Resources Division of Geology and Land Survey

LOCATION: $\quad$ Statewide

PROJECT CHIEF: L. D. Hauth

PROBLEM: $\quad$ As population increases and industry expands, the demands for water increase. Water use, such as irrigation, has placed greater demands on both ground and surface waters. Thus effective water-resources management is needed. Basic to that need is determining how much water is being used. The collection and management of water-use information is complex and requires careful planning. Data must be collected and stored so that analysis, inspection of data, and accuracy goals can be met. Therefore, a we11-planned storage and retrieval system is necessary before collection of water-use data.

OBJECTIVES: Plan a water-use data-collection and management program for Missouri. The planning will assure that data collected, as well as its format, is compatible with the National Water-Use Data System (NWUDS), and meets national and State objectives. The planning phase will assure that the maintenance and dissemination of data on current water uses will allow projections on future water use. 
A task force committee will be selected from among State, Federal, local, municipal, and private agencies, which have an interest in water-use information in Missouri, to meet and allow input into the data system. A determination of available data for inclusion in the data base will be made. Requirements for the functions, software/hardware, and interface to the NWUDS will then be documented.

PLANS FOR

NEXT YEAR:

PLANNED

REPORT :
Complete and document the plan for implementation of a data-collection, storage, and retrieval system for a water-use data base.

Missouri water-use data system documentation. Will be published as a State open-file report.

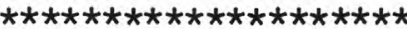

PROJECT TITLE: Hydrology of Streams in St. Louis County MD 70-017 COOPERATOR: St. Louis County, Department of Highways and Traffic LOCATION: $\quad$ St. Louis County, Missouri

PROJECT CHIEF: T. W. Al exander

PROBLEM: $\quad$ As the population of St. Louis City declines, the population of St. Louis County increases. Substantial changes are being made in the use of land in the rural areas. Stormwater drainage is one of the principal problems. Inadequate data for planning of roads, use of flood-plain areas, zoning ordinance and channel improvements necessitate collection of data needed for flood-inundation maps and other analyses.

OBJECTIVE: $\quad$ To determine the effects of urbanization on runoff from small streams in St. Louis County. Generate frequency profiles to be displayed on a base map compatible to the users need $(1: 6,000$ scale).

APPROACH: $\quad$ Reconnaissance of streams, which drain the areas that will be affected by urbanization, will determine the number of data-collecting sites needed for definition of the hydrologic characteristics of the area. Flood and other flow data will be collected at many sites to define flood frequencies, flood profiles, channel shapes and capacities, flow characteristics, and other factors needed to study the suburban-rural hydrology. Gaging stations, crest-stage gages, and precipitation stations have been installed. 
PROGRESS AND

SIGNIF ICANT

RESULTS:

PLANS FOR

NEXT YEAR:

REPORTS :
The 16 basins having 100-year flood profiles are being completed and the 1:6,000 scale maps delineating the area inundated by the 100-year flood are. in the final phase of completion. A flood-frequency report documenting the results and conclusions reached in the analys is of all the data has been published as a Water-Resources Investigations report.

Continuation of the rainfall-runoff network to monitor changes brought about by urbanization.

Spencer, D. W., and Alexander, T. W., 1979, Techniques for estimating the magnitude and frequency of floods in St. Louis County, Missouri: U.S. Geological Survey Water-Resources Investigations 78-139, 23 p.

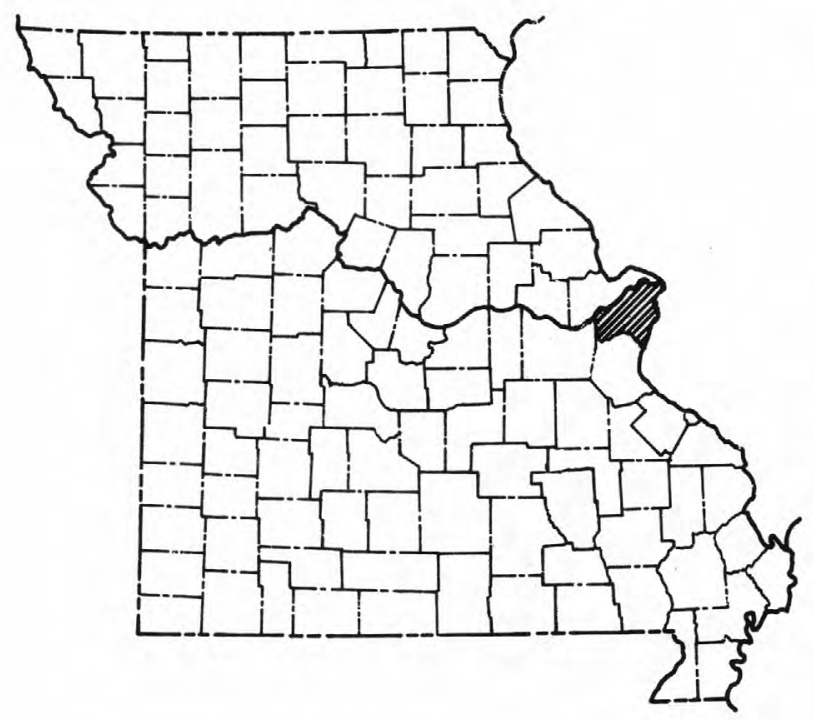


PROJECT TITLE: Flood Hazard Information, House Document 465

MØ 73-025

LOCATION: $\quad$ Statewide

PROJECT CHIEF: L. D. Hauth

PROBLEM: $\quad$ House Document 465 outlines a national program to provide flood-hazard information. The Geological Survey has been assigned the responsibility to outline on existing USGS topographic maps those flood-prone areas that can be identified from information on the maps and from flood-frequency studies previously made.

OBJECTIVE: $\quad$ To identify and label on USGS topographic quadrangle maps the flood-prone areas of cities and towns, over 2,500 population, and adjacent areas for which adequate maps are available and flood frequency-drainage area relationship can be determined.

APPROACH: In general, project requirements will be met using information already available. Where possible, relationships between flood depth, flood discharge, frequency of occurrence, and drainage area will be used to define flood profiles and flood boundaries (100-year) along streams shown on topographic maps, but for which in many cases no actual flood information exists. Regional flood depth-frequency relationships will be used where they can be identified.

PROGRESS AND No maps published in 1978 fiscal year.

SIGNIFICANT

RESULTS:

PLANS FOR

NEXT YEAR:

Flood-prone area maps for Vienna, Doniphan, Piedmont, Warrenton, and Wright City quadrangles. 
PROJECT TITLE: Water resources of the Southeastern Lowlands Mø 75-029

COOPERATOR: Department of Natural Resources

Division of Geology and Land Survey

LOCATION: $\quad$ Southeast Missouri

PROJECT CHIEF: R. R. Luckey

PROBLEM:

The Southeastern Lowlands is the largest irrigated agricultural area in Missouri. The irrigated acreage increased from 1,550 acres in 1949 to over 150,000 acres in 1975 . The area is suitable for additional agricultural development. Quantitative data on the characteristics of the alluvial aquifer, and information on the degree of the present development must be collected to evaluate the effects of anticipated additional development. Evaluation of the amount of water available for future development must be complemented by an evaluation of the quality of water in the aquifer and the susceptibility of the aquifer to pollution.

OBJECTIVE: Determine the amount of ground water in storage in the alluvial aquifer. Investigate the location, source, and amount of natural recharge and discharge. Collect information on the present rate and location of ground-water withdrawal and estimate the effects of probable future withdrawal patterns. Determine the relationship between the alluvial aquifer and the natural and manmade drainage systems. Collect the quantitative data that will most likely be needed in future model studies of the area. Describe the chemical quality of both the surface water and the ground water. Update previous low-flow frequency and flow-duration analyses.

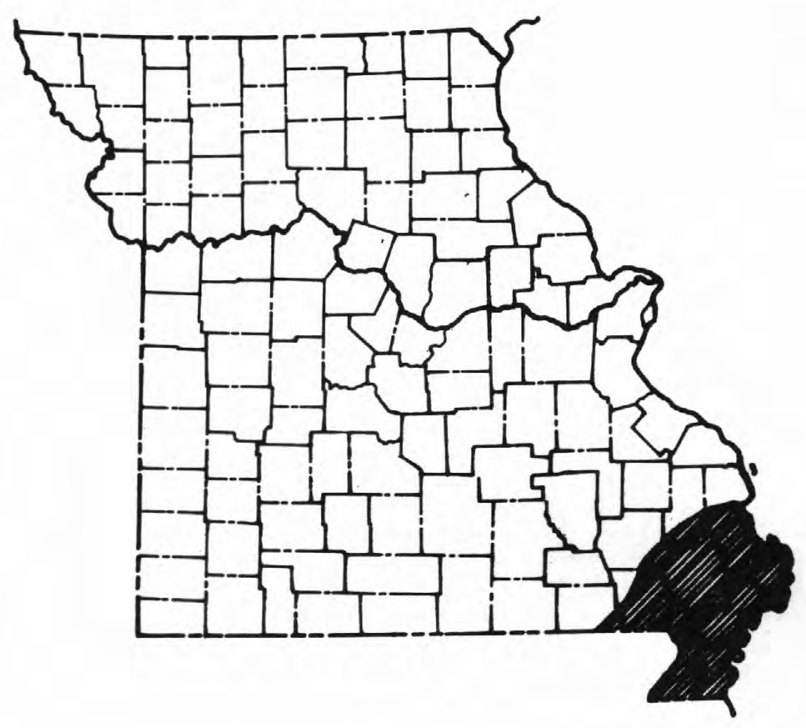


Water resources of the Southeastern Lowlands MØ 75-029--continued

PROGRESS AND

SIGNIFICANT

RESULTS:

PLANS FOR

NEXT YEAR:
All field work has been completed and the hydrologic data and interpretive reports are in review.

Complete hydrologic data and interpretive reports and receive approval for publication.

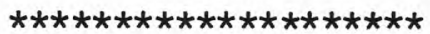

PROJECT TITLE: Hydrology of Osage Fork, Grandglaize, and Niangua River basins, Missouri Mø 75-030

COOPERATOR: Department of Natural Resources

Division of Geology and Land Survey

LOCATION: $\quad$ Central Missouri

PROJECT CHIEF: John Skelton

PROBLEM: $\quad$ Numerous streams in the carbonate terranes of the Missouri 0zarks lose water to the ground-water system, which is used extensively as the source for water supplies. In areas where losing streams exist, control of development is needed to insure that ground water is not contaminated through recharge via these losing stream segments. In order for the responsible officials to control development properly and manage the land resource wisely, they need information not now available that is indicative of areas where pollution could originate through unwise location of waste-disposal facilities or accidental spill.

OBJECTIVE: The general objective of the study is to present hydrologic information on the 0sage Fork, Niangua River, and Grandglaize Creek basins, emphasizing losing streams and their interrelation with aquifers.

Specific objectives are as follows:

1. Determine the low-flow characteristics of all streams in enough detail to implement water-quality regulations.

2. Evaluate methods that will lead to better definition of losing streams and basins having deficient runoff. 
3. Provide guidelines for relating the flow system to land use, vegetation distribution, soil and physiography when transferring relationships to other humid regions of carbonate rocks.

APPROACH :

(1) Seepage runs will be made to form the base for detailed studies of low-flow characteristics. The distribution of anomalous and normal streamflow patterns will be related to geologic data. (2) Types of vegetation as they relate to moisture in the valley will be described. (3) Potentiometric maps necessary to describe the flow system will be drawn. (4). Subsurface geologic mapping will be done to relate stratigraphy, lithology, and structure to ground-water levels. (5) Water quality of wells and springs will be determined to aid in locating recharge areas. (6) Earth Resources Technology Satellite (ERTS) imagery will be used to relate vegetation, land use, and physiography to runoff.

PROGRESS AND SIGNIFICANT RESULTS:

PLANS FOR NEXT YEAR:

REPORTS:
The final report was completed and is now in final review.

Reviews will be completed, and the report will be delivered to the cooperator.

Skelton, John, and Miller, D. E. 1978, Tracing subterranean flow of sewage-plant effluent in Lower Ordovician Dolomite in the Lebanon area, Missouri. [Accepted by Ground Water for publication.]

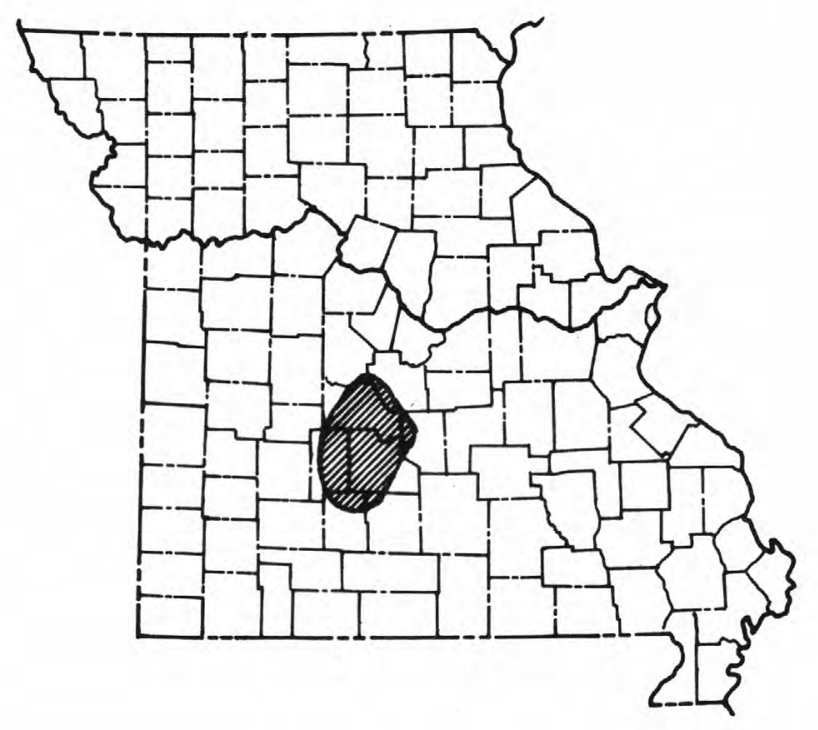


Hydrology of Osage Fork, Grandglaize, and Niangua River basins Mø 75-030--continued

REPORTS: Harvey, E. J., and Skelton, John, 1978, Relationship between hydrology and bottomland vegetation in the Ozarks Mountains of Missouri: U.S. Geological Survey Journal of Research, v. 6, no. 3, p. 299-305.

Harvey, E. J., Skelton, John, and Miller, D. E., 1978, Hydrology of carbonate terrane - The Niangua River, Osage Fork, and Grandglaize Creek basins, Missouri: Missouri Division of Geology and Land Survey, Water Resources Report. [In review.]

\begin{tabular}{|c|c|}
\hline PROJECT TITLE: & Water for irrigation in Audra in County, Missouri Mø 78-032 \\
\hline COOPERATOR: & $\begin{array}{l}\text { Department of Natural Resources } \\
\text { Division of Geology and Land Survey }\end{array}$ \\
\hline LOCATION: & Northeastern Missouri \\
\hline PROJECT CHIEF : & L. F. Emmett \\
\hline PROBLEM: & $\begin{array}{l}\text { Water for supplemental irrigation in Audrain County is } \\
\text { from surface reservoirs and deep wells. The surface } \\
\text { reservoirs generally impound less than 100-acre-feet, } \\
\text { consequently they may not be full in dry years. Because } \\
\text { an apparent interface between fresh and saltwater in } \\
\text { the deep aquifer extends through northern Audrain County, } \\
\text { there is concern that increased pumping of ground water } \\
\text { could cause water quality to deteriorate in some places. } \\
\text { There are needs to determine the quantity and quality of } \\
\text { water available for irrigation, and to monitor the effects } \\
\text { of increased withdrawal of ground water from the deep } \\
\text { aquifer. }\end{array}$ \\
\hline OBJECTIVE: & $\begin{array}{l}\text { Evaluate the total water resource of Audrain County in } \\
\text { order to determine the availability and quality of water } \\
\text { for irrigation. Determine the potential for saltwater } \\
\text { encroachment as a result of pumping from the deep aquifer. }\end{array}$ \\
\hline
\end{tabular}


APPROACH: $\quad$ Compilation of hydrogeologic information present in the files and in the literature. Field inventory of wells in Audrain County with emphasis on locating wells on the saltwater side of the interface. Collection of water-use, water-level, water-quality, and aquifer characteristics data to supplement file data. Compilation of file data to define surface-water characteristics will be augmented by reconnaissance of streams during a low-flow period to document low-flow characteristics.

PROGRESS AND SIGNIFICANT RESULTS:

PLANS FOR NEXT YEAR:

REPORTS :

Inventoried and measured water levels in about 110 wells. Entered well data into System 2000. Constructed potentiometric map of deep aquifer. Collected and analyzed water samples from 15 wells.

Complete well inventory. Measure water levels in wells; mass measurement before and after pumping. Also periodic (bi-weekly to monthly) measurement in selected wells. Construct potentiometric maps--before and after irrigation season. Collect data on water use. Determine regional transmissivity from flow-net analys is. Computer simulation of deep aquifer. Collect and analyze water samples from wells.

Planned: Annual Progress Report.

Interpretative report on completion of project.

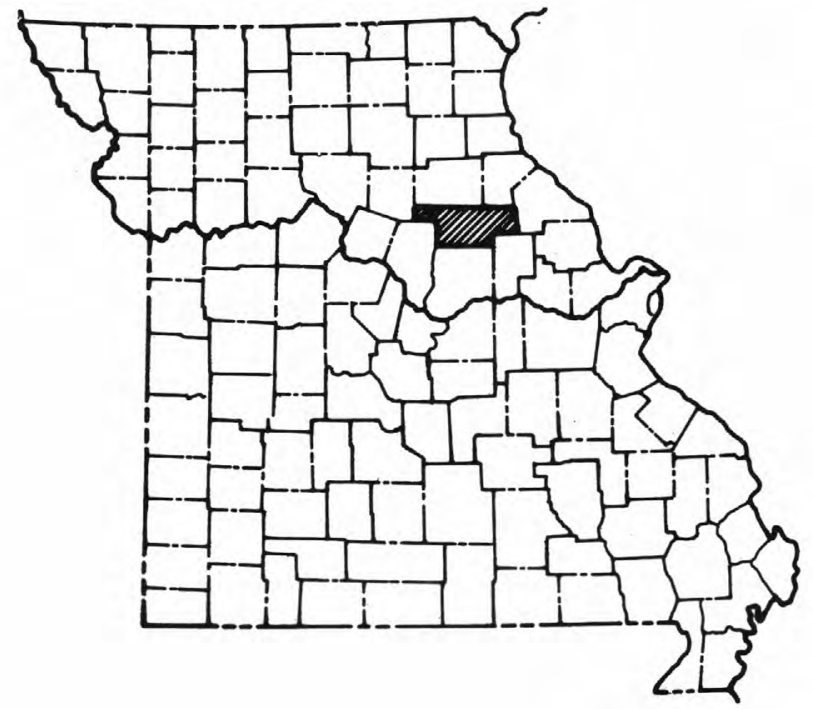


Table 5.--Water-quality station network, 1979

[Type of station: $C=$ Chenical; $S=$ Sediment; $T=$ Temperature. Source of funding: DEQ, Division of Environmental Quality; Mo. Conserv. Comm., Mis souri Conservation Commission; City of Spfd., City of Springfield; USGS, U.S. Geological Survey; NPS, National Park Service; CE KC, Corps of Engineers, Kansas City; CE STL, Corps of Engineers, St. Louis; CE RI, Corps of Engineers, Rock Island; CE TU, Corps of Engineers, Tulsa; CE LR, Corps of Engineers, Little Rock.]

\begin{tabular}{|c|c|c|c|c|c|c|c|c|c|c|c|c|}
\hline & & & & & & SOURCE & OF FUN & ING & & & & \\
\hline $\begin{array}{c}\text { Station } \\
\text { no. }\end{array}$ & Station name & $\begin{array}{l}\text { Type } \\
\text { of } \\
\text { record }\end{array}$ & DEQ & $\begin{array}{l}\text { Mo. } \\
\text { Conserv. } \\
\text { Comm. }\end{array}$ & $\begin{array}{l}\text { City } \\
\text { of } \\
\text { Spfd. }\end{array}$ & USGS & NPS & CE KC & CE STL & CE RI & CE TU & CE LR \\
\hline 05490600 & Des Moines River at St. Francisville---- & CST & $x$ & $-\cdots-$ & --- & $x$ & --- & $-\cdots-$ & -.-.- & -...-- &.---- & -...- \\
\hline 05508000 & Salt River near New London- & CST & $x$ & $-\cdots$ & --- & $x$ & --- & $-\cdots-$ & -...- & 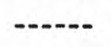 & -...... & -...- \\
\hline 05587550 & Mississippi River below Alton, Ill-..... & CST & $x$ & $-\cdots-$ & --- & $x$ & --- & $-\cdots--$ & $-\cdots-$ & $-\cdots-$ & ---- & -..-- \\
\hline 06817800 & Nodaway River near Oregon-1- & C & $x$ & $-\cdots$ & $\cdots$ & $x$ & --- & $-\cdots--$ & ----- & ---- & $-\cdots$ & $-\cdots--$ \\
\hline 06818000 & Missouri River at St. Joseph & CST & $x$ & ---- & --- & $x$ & --- & $-\cdots$ & $-\cdots$ & $-\cdots$ & $-\cdots-$ & $-\cdots$ \\
\hline 06821190 & Platte River at Sharps Station & CST & -- & $-\cdots-$ & $-\cdots$ & $x$ & $-\cdots$ & $-\cdots-$ & -....- & -....- & -..... & \\
\hline 06902000 & Grand River near Sumner & CST & $x$ & $-\cdots$ & $-\cdots$ & $x$ & -- & $-\cdots-$ & $-\cdots--$ & $-\cdots-$ & $-\cdots-$ & \\
\hline 06905500 & Chariton River near Prairie Hill & CST & --- & $-\cdots$ & $-\cdots$ & $x$ & -- & $-\cdots-$ & $-\cdots--$ & - - - & $-\cdots--$ & \\
\hline 06908800 & Lamine River near Blackwater & CST & -- & -.-.- & $-\cdots$ & $x$ & --- & $-\cdots--$ & ----- & $-\cdots--$ & $-\cdots--$ & \\
\hline 06916650 & Marais Des Cygnes River near Worland---- & C & $x$ & $----\cdot$ & $-\cdots$ & $x$ & -- & $-\cdots--$ & $-\cdots--$ & $-\cdots-$ & $-\cdots--$ & \\
\hline 06918080 & Osage River near Schell City-............... & CST & $-\cdots$ & $-\cdots$ & $\cdots$ & $x$ & --- & $-\cdots--$ & $-\cdots--$ & $\cdots--$ & $-\cdots--$ & \\
\hline 06918440 & Sac River near Dadeville--..-- & $T$ & $-\cdot$ & $-\cdots-$ & $-\cdots$ & $-\cdots$ & -- & $x$ & ----- & $\cdots--$ & $-\cdots--$ & \\
\hline 06918990 & Stockton Lake near Stockton--.-- & $T$ & -- & $-\cdots$ & --- & $-\cdots$ & --- & $x$ & $-\cdots--$ & $-\cdots--$ & $-\cdots--$ & $-\cdots-$ \\
\hline 06921350 & Pomme de Terre River near Hermitage-....- & $T$ & --- & $-\cdots$ & $-\cdots$ & $-\cdots$ & --- & $x$ & $-\cdots--$ & $-\cdots--$ & ----- & $-\cdots--$ \\
\hline 06921500 & Porme de Terre River at Hermitage------- & $T$ & --- & $-\cdots$ & --- & $-\cdots$ & -- & $x$ & $-\cdots--$ & $-\cdots--$ & $-\cdots--$ & $-\cdots-$ \\
\hline 06922200 & Tebo Creek at Leesville-1 & C & --- & $-\cdots$ & $-\cdots$ & $\cdots$ & $\cdots$ & $x$ & - - - & $-\cdots-$ & $\cdots-\cdots$ & $\cdots$ \\
\hline 06922500 & 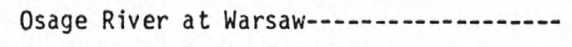 & $T$ & -- & $-\cdots$ & --- & $-\cdots-$ & --- & $x$ & $-\cdots-$ & ---- & $-\cdots--$ & $-\cdots-$ \\
\hline 96926510 & Osage River below St. Thomas & CST & $x$ & $-\cdots$ & $-\cdots$ & $x$ & -- & $-\cdots-$ & $-\cdots$ & $-\cdots$ & $-\cdots-$ & $-\cdots$ \\
\hline 06928600 & Gasconade River near Hooker-........- & c & $x$ & ---- & --- & $x$ & --- & ---- & ----- & ----- & ----- & $-\cdots-$ \\
\hline 06930450 & Big Piney River at Devils Elbow-.....- & C & $x$ & $-\cdots$ & $\cdots$ & $x$ & -- & $-\cdots-$ & $-\cdots-1$ & $-\cdots$ & $-\cdots$ & - \\
\hline 06930800 & Gasconade River above Jerome-... & CST & $x$ & $-\cdots$ & $-\cdots$ & $x$ & -- & 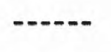 & $-\cdots-$ & $-\cdots$ & ---- & \\
\hline 06934500 & Missouri River at Hermann & CST & & $-\cdots$ & $\cdots$ & $x$ & $\cdots$ & $-\cdots-$ & ---- & ----- & $-\cdots$ & \\
\hline 07010000 & Mississippi River at St. Lotiss- & ST & $\cdots$ & $\cdots$ & $-\cdots$ & $x$ & -- & $-\cdots--$ & $x$ & $-\cdots--$ & $-\cdots--$ & \\
\hline 07014500 & Meramec River near Sullivan-_........... & C & $x$ & --- & $-\cdots$ & $x$ & -- & $-\cdots-$ & $-\cdots-$ & $-\cdots-1$ & - - - - & \\
\hline 07019000 & Meramec River near Eureka-...- & CST & $\cdots$ & --- & $-\cdots$ & $x$ & $\cdots$ & $\cdots-$ & $-\cdots-$ & $-\cdots-$ & - n & $-1-1$ \\
\hline 07022000 & Mississippi River at Thebes, III--- & CST & $x$ & $-\cdots$ & --- & $x$ & --- & $-\cdots-$ & $-\cdots$ & $-\cdots-$ & ----- & ---- \\
\hline 07046001 & Little River ditches near Kennett--.--- & C & $x$ & --- & 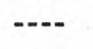 & $x$ & $-\cdots$ & ---- & ---- & $-\cdots-$ & $-\cdots--$ & ---- \\
\hline 07050750 & James River near Nixa-- & $T$ & $x$ & $-\cdots$ & --- & $x$ & $-\cdots$ & $-\cdots$ & --- & $-\ldots$ & 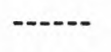 & ---- \\
\hline 07057600 & James River near Wilsons Creek-_----- & $c$ & $x$ & $\ldots$ & $-\cdots$ & $x$ &.-- & $-\cdots$ & -...- & 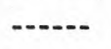 & $\ldots$ & \\
\hline
\end{tabular}



PROJECT TITLE: An assessment of water quality in the proposed Prosperity Reservoir area, Center Creek basin, Missouri Mø 78-033

COOPERATOR: Corps of Engineers, Tulsa District

\section{LOCATION: $\quad$ Southwest Missouri}

PROJECT CHIEF: J. H. Barks

PROBLEM:

The Corps of Engineers is in the preconstruction planning phase for the proposed Prosperity Dam and Reservoir on Center Creek in southwest Missouri. One step in their planning process is an assessment of existing water quality, and they have requested the U.S. Geological Survey to conduct the water-quality study.

OBJECTIVE: $\quad$ To provide an assessment of existing water quality in the reservoir area, to define potential water-quality problems associated with the proposed reservoir project, such as pollution from abandoned zinc and lead mines and municipal and industrial wastes.

APPROACH: $\quad$ Review previous water-quality studies on the Center Creek watershed for information pertinent to this study. Four sets of samples will be collected during base-flow conditions and a storm event will be sampled during the rise, peak, and recession at two sites at the upstream end, and one site at the downstream end of the study area. These samples will be analyzed for major inorganics, trace elements, pesticides, and nutrients. One bed material sample from each site will be analyzed for trace elements.

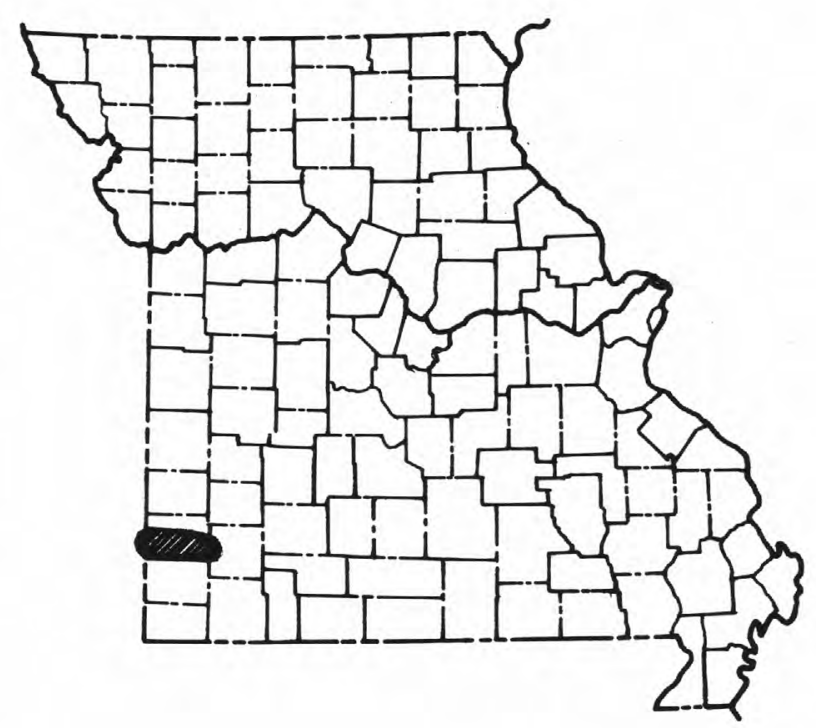


An assessment of water quality in the proposed Prosperity Reservoir area, Center Creek basin, Missouri MØ 78-033--continued

PROGRESS AND SIGNIFICANT RESULTS:

PLANS FOR NEXT YEAR:

REPORTS :
All data collection was completed. The data were analyzed and an interpretive report was prepared and published. Water that would fill the proposed reservoir is of good quality, but nutrient concentrations are probably high enough to cause the lake to become eutrophic.

Work on this project has been completed. This project has been extended to allow more time for data to accumulate. A report describing the additional data and interpretations will be prepared.

Barks, J. H., and Berkas, W. R., 1979, Water quality in the proposed Prosperity Reservoir area, Center Creek basin, Missouri: U.S. Geological Survey Water-Resources Investigations $79-22,26 \mathrm{p}$.

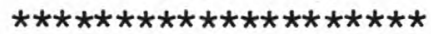

PROJECT TITLE: Ground-water appraisal of Prosperity Reservoir area, Center Creek basin, Missouri MØ 78-034

COOPERATOR: Corps of Engineers, Tulsa District

LOCATION: $\quad$ Southwest Missouri

PROJECT CHIEF: E. J. Harvey

PROBLEM:

OBJECTIVE :
The Corps of Engineers is in the preconstruction planning phase of the proposed Prosperity Dam and Reservoir on Center Creek in southwestern Missouri. One step in their planning process is an evaluation of present and potential ground-water supplies in the area. The Corps of Engineers has requested the U.S. Geological Survey to do the study.

(1) Evaluate present and potential water supply from Ordovician and Cambrian aquifers, (2) determine storage of water in enclosing Mississippian rocks, (3) evaluate chance of leakage past the damsite and downstream change in ground-water levels, and (4) determine whether or not ground-water discharge from mined areas could affect water quality in the reservoir or in Center Creek. 
(1) Evaluate available ground-water data, (2) supplement available data with field data collection in critical areas. Include seepage runs to correlate increase in discharge of Center Creek at Oronogo-Duenweg belt with a change in potentiometric surface, and (3) estimate storage characteristics of the Mississippian limestone in the Oronogo-Duenweg belt and in the area of the reservoir.

PROGRESS AND SIGNIFICANT RESULTS:

PLANS FOR NEXT YEAR:

REPORTS :

A preliminary report was completed, which included results from a model of the ground-water system in the reservoir area. The model showed potential rises of 20 feet near the reservoir, and 0.5 to 1.0 foot in the southern part of Grove Creek basin. Grove Creek, immediately below the proposed damsite, is a losing stream. It is possible that flow from the losing reach can enter caverns, which might conduct the water to the Oronogo-Duenweg mining belt. A rise in water level in the upland adjacent to the losing reach of Grove Creek could also cause water to move toward the mining belt.

(1) Trace dye from losing reach to Scotland Spring on Grove Creek, (2) make seepage runs on Grove Creek and on Center Creek upstream and downstream from mouth of Grove Creek, (3) add a few additional control points in Mississippian limestone near damsite, and (4) set up monitoring network of observations wells and shafts in reconnaissance area.

Harvey, E. J., and Emmett, L. F., 1978, Hydrology and mode1 study of the proposed Prosperity Reservoir, Center Creek basin, southwestern Missouri: U.S. Geological Survey Water-Resources Investigations. [In review.]

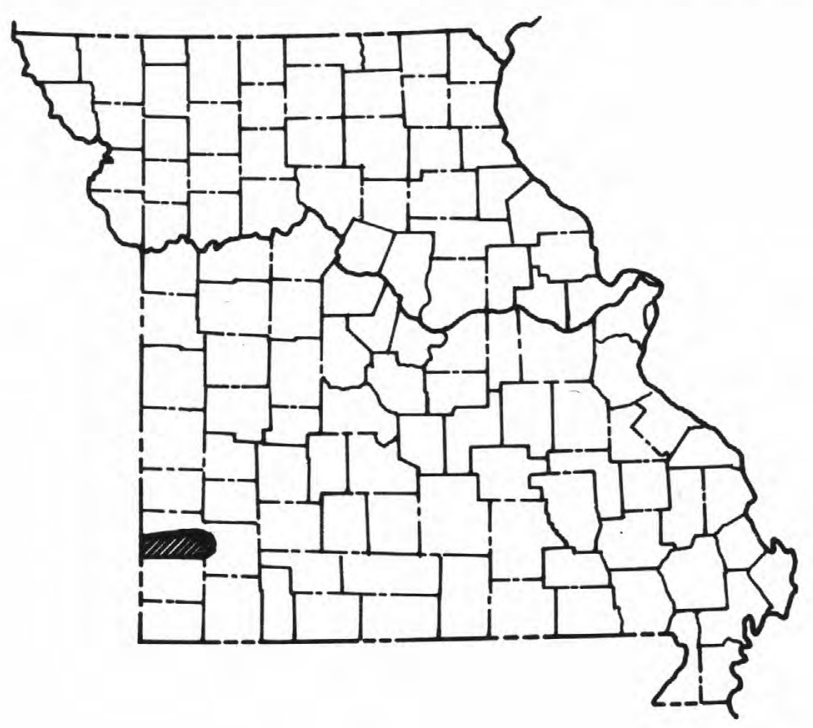


PROJECT TITLE: Effects of urban runoff and wastewater effluent on Wilsons Creek and James River near Springfield, Missouri Mø 78-035

COOPERATOR: $\quad$ City of Springfield, Missouri

LOCATION: $\quad$ Southwestern Missouri, Christian, and Greene Counties

PROJECT CHIEF: John Skelton

PROBLEM: $\quad$ The quality of water in Wilsons Creek and James River has been degraded by effluent from the Southwest Wastewater

Plant and storm runoff from streets, parking lots, and industrial areas. Rainfall, streamflow, and water-quality data collected prior to upgrading the Southwest Wastewater Plant (October 1977) need to be summarized and interpreted to show changes in quality of water in Wilsons Creek and James River with respect to effluent discharge, rainfall, runoff, and time.

OBJECTIVE: $\quad$ Summarize and interpret rainfall, streamflow, and waterquality data collected from Wilsons Creek and James River before the new wastewater plant went into operation (through September 1977). This report will serve as a base from which future changes in the quality of water in Wilsons Creek and James River can be determined.

APPROACH: $\quad$ Samples for physical properties, common inorganic constituents, major nutrients, and bacteria will be collected monthly from the James River upstream and downstream from Wilsons Creek and analyzed to show the effects of Wilsons Creek on James River. Continuous discharge, water temperature, specific conductance, dissolved oxygen, and $\mathrm{pH}$ data collected for Wilsons Creek upstream and downstream from the Southwest Wastewater Plant, and the James River downstream from Wilsons Creek will be analyzed to show progressive downstream changes caused by effluent discharge and storm runoff. Continuous rainfall and discharge from several sites will be correlated with water-quality data and used to evaluate the possibilities of estimating peak flows and flood volumes.

PROGRESS AND Available data were compiled and interpretation began SIGNIFICANT RESULTS: during fiscal year 1978.

PLANS FOR NEXT YEAR:

Complete a Water-Resources Investigations/NTIS report and receive Director's approval. 
REPORTS:

Effects of urban runoff and wastewater effluent on Wilsons Creek and James River near

Springfield, Missouri. [In review.]

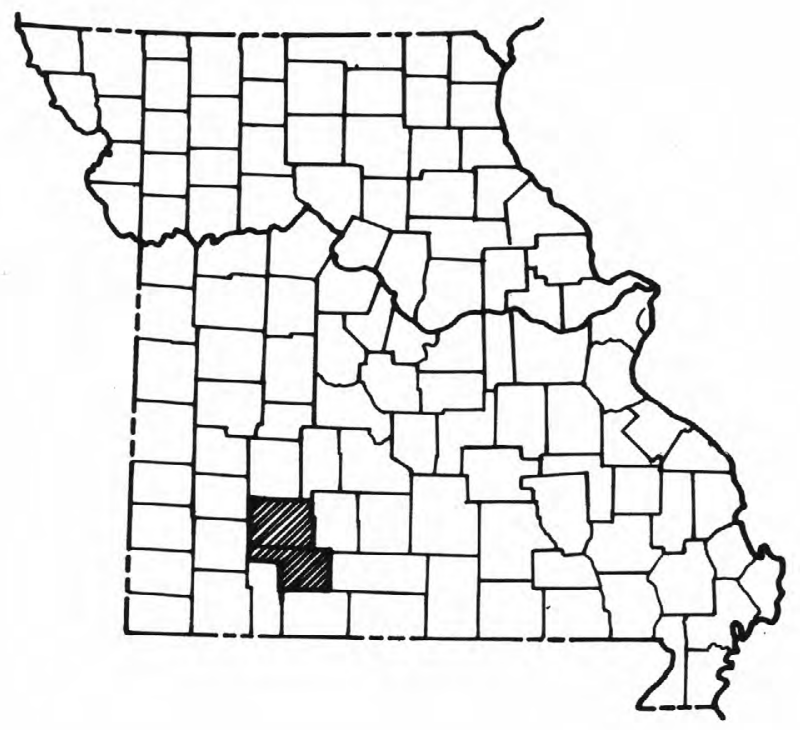


PROJECT TITLE: Regional hydrogeology of aquifers of Cambrian and Ordovician age in parts of Minnesota, Wisconsin, Iowa, Illinois, Indiana, and Missouri Mø 79-036

PROJECT CHIEF: Walter Steinhilber, Madison, Wisconsin

Project coordinator in Missouri--Leo F. Emmett

LOCATION: $\quad$ Northern Missouri and other states.

PROBLEM: $\quad$ The northern Midwest is underlain by a continuous aquifer system formed of rocks of Paleozoic age. This system suppl ies water for municipal, agricultural, industrial, and domestic uses. Increasing use of water has caused the public to be concerned about the effects of pumping from the system and about the availability of water supplies. The problems causing public concern can be better solved if there is an understanding of how withdrawals at one place may affect the uses of water at another place. The system is poorly understood on a regional scale partly because not all hydrologic facts have been related to each other by a conceptual model.

OBJECTIVE: $\quad$ To provide regional concepts that will allow evaluation of the aquifer's water-supply potential and its response to projected development schemes. More specifically the study will be designed to: (1) provide data that helps describe the geologic, hydrologic, and chemical quality of the aquifer system, (2) estimate past and future withdrawals from the aquifer system and determine present withdrawals, and (3) develop a conceptual model of the aquifer system.

APPROACH: In cooperation with the Missouri Division of Geology and Land Survey compile and evaluate selected data as they may relate to regional flow systems. Resultant concepts and data may then be used to select logical hydrologic boundaries for a digital-computer model of the regional flow system. The model might then be used to test the validity of the concepts and for its predictive capability. 
PLANS FOR NEXT YEAR:

REPORTS :
Data from the files of the Missouri Division of Geology and Land Survey and U.S. Geological Survey will be compiled, coded, and used to construct maps showing:

1. Water-level and water-bearing characteristics of selected aquifers.

2. Water quality of selected aquifers.

3. Structure contours on tops of Cambrian-Ordovician aquifer; Mississippian aquifer and Lamotte Sandstone; isopachous maps of Cambrian-Ordovician aquifer and Lamotte Sandstone.

A field reconnaissance to locate and inventory selected deep wells will begin.

Planned: Water-Resources Investigations report, or bulletin of the Missouri Division of Geology and Land Survey.

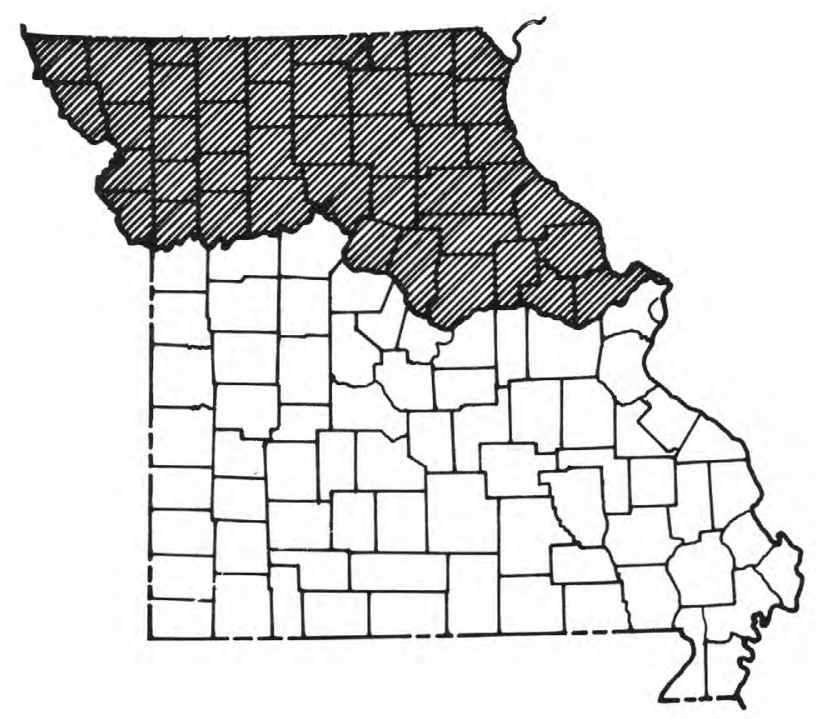


PROJECT TITLE: Water for energy development, agriculture, and industry in northwestern Missouri $M \emptyset$ 79-037

COOPERATION: Department of Natural Resources Division of Geology and Land Survey

LOCATION: $\quad$ Northwestern Missouri

PROJECT CHIEF: John Skelton

PROBLEM:

OBJECTIVE: (1) Del imit areas of northwestern Missouri where water demand will be accelerating because of increasing irrigation, municipal, and industrial development, and assess water resources potential in these areas, (2) assemble detailed information about the location of active and potential coal mining and describe in as much detail as possible the hydrology in these areas, (3) based on information from (1) and (2), recommend areas for future detailed hydrologic investigations by the U.S. Geological Survey and the Division of Geology and Land Survey in northwestern Missouri.

APPROACH: Define areas in northwestern Missouri where water demand will be accelerating because of increasing agricultural and industrial developments. Identify areas where significant coal strip-mining will be occurring in the next 20 to 30 years. On a limited basis, inventory wells and measure water levels in areas defined so that generalized potentiometric maps can be prepared. Make seepage runs on selected large tributary streams if low-flow conditions permit. Compile hydrologic data for areas defined and evaluate needs for further data collection and analyses.

PLANS FOR NEXT YEAR:

Through literature search and contacts with State and Federal agencies, define areas where water demand will increase in the future. Identify areas of present and future significant coal strip-mining. Inventory and measure water 1evels for 1 imited number of wells. Make seepage runs on large tributary streams if low-flow conditions are favorable. Begin compilation of data and prepare outline and introductory sections of report. 
REPORTS: Planned: Water for energy development, agriculture, and industry in northwestern Missouri, Water-Resources Investigations series.

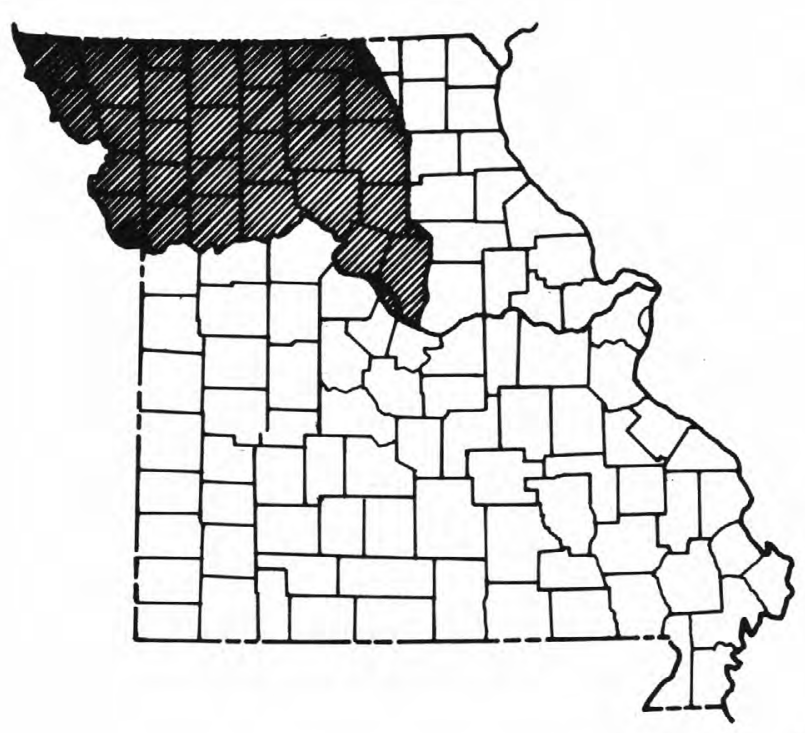


Hydrologic and Water-Quality Characteristics of Streams and Shallow Aquifers in Coal-Mining Areas of Missouri

To administer regulations set forth in the Surface Mining Control and Reclamation Act of 1977, hydrologic information will be needed to classify streams and define water quantities and quality in streams and shallow aquifers. A project has been proposed to the II.S. Geological Survey to classify coal-mining areas into subareas having similar geologic, topographic, and hydrologic characteristics; relate streamflow parameters to basin parameters in the subareas; characterize water quality in the subareas and relate these parameters to streamflow or geologic parameters; and to evaluate changes in water quantity and quality in selected areas.

\section{$\star \star \star * * * \star * \star * * * * \star * \star * \star * \star *$ \\ Regional Hydrogeology of Aquifers of Paleozoic Age in Parts of Arkansas, Missouri, Kansas, Oklahoma, and Texas}

The central Midwest is underlain by a continuous aquifer system that supplies fresh water in Arkansas and Missouri, but has been used for waste disposal in other states. The operation of the flow system in this aquifer is poorly understood, and many studies have not been hydrologically related to one another.

A regional study of the aquifer system has been proposed to include the central Midwest states. Planning for the study will begin in fiscal year 1979 with the cooperation of the Division of Geology and Land Survey. The study will be designed to provide information necessary to understand flow in the system, provide a framework to interrelate hydrologic studies of smaller areas and to plan detailed studies of smaller areas, and to evaluate the consequences of stresses that have or can be assumed to be placed on the aquifer.

\section{Urban Hydrology in St. Louis County}

Flood frequency and areas inundated by the 100-year flood have been defined in much of the county. Water-quality characteristics, however, are not generally defined; yet, contamination from non-point sources can have serious consequences in receiving streams and lakes. A project is being proposed in cooperation with the East-West Gateway Coordinating Council to provide water-quality data from a basin in the county; relate selected land uses and environmental practices in the basin to water quality during selected runoff events; and to evaluate selected water-quality models. 
Selected references on water resources in Missouri are listed on the following pages; many of them are available for inspection at the office of the U.S. Geological Survey and the Division of Geology and Land Survey in Rolla. New reports published by the Geological Survey are announced monthly in "New Publications of the Geological Survey." Subscriptions to this monthly list are available on request to the U.S. Geological Survey, 329 National Center, Reston, Va. 22092.

Professional papers, water-supply papers, bulletins, and the Geological Survey's annual report are sold by the U.S. Geological Survey, Branch of Distribution, 1200 South Eads Street, Arlington, Va. 22092 (authorized agent of the Superintendent of Documents, Government Printing Office). Circulars are free upon request to the U.S. Geological Survey, National Center, Reston, Va. 22092. Water-resources investigations folders for each of the 50 States and Puerto Rico also are free upon request. Hydrologic investigations atlases, hydrologic unit maps, and other maps pertaining to Missouri are sold by the U.S. Geological Survey, Branch of Distribution, Bldg. 41, Denver Federal Center, Lakewood, Colo. 80225.

Records of streamflow, ground-water levels, and quality of water have been published for many years as Geological Survey Water-Supply Papers (WSP). Beginning with the 1975 water year, however, this series was replaced by a new publication series, U.S. Geological Survey Water-Data Reports. This new series combines under one cover for each state: streamflow data, water-qual ity data for surface and ground water, and ground-water level data from the basic network of observation wells. For Missouri the title is, "Water-Resources Data for Missouri - Water Year 1978: U.S. Geological Survey Water-Data Report M0-78-1." Other publications series of general interest also are explained in the following pages. Further information on these publications can be obtained from the District Chief, Water Resources Division, Rolla, Mo. 65401

A more complete State List of reports of the U.S. Geological Survey is given in the bibliography. Summary statements about the immediate national water situation are presented in the "Water Resources Review," which is issued monthly. The Review may be obtained free on application to the U.S. Geological Survey, 420 National Center, Reston, Va. 22092.

Indexes to the "Catalog of Information on Water Data" are available free from the District Chief in Rolla, or the Office of Water Data Coordination, U.S. Geological Survey, 417 National Center, Reston, Va. 22092. 
Open-file reports are available for inspection at the office from which the report originated. Flood-prone area maps may al so be obtained from the Missouri district office.

The Geological Survey National Center maintains a library with an extensive earth-sciences collection. Local libraries may obtain books, periodicals, and maps through interlibrary loan by writing to: U.S. Geological Survey Library, 12201 Sunrise Valley Drive, Reston, Va. 22092. 


\section{BIBLIOGRAPHY}

PROFESSIONAL PAPERS (PP)

448-A. Availability of water in the Mississippi Embayment, by E. M. Cushing and others. 1970.

448-B. General geology of the Mississippi Embayment, by E. M. Cushing, E. H. Boswell, and R. L. Hosman. 1964.

448-C. Cretaceous aquifers in the Mississippi Embayment, by E. H. Boswell and others. 1965.

448-D. Tertiary aquifers in the Mississippi Embayment, by R. L. Hosman and others. 1968.

448-E. Quaternary aquifers in the Mississippi Embayment, by E. H. Boswell and others. 1968.

448-F. Low-flow characteristics of streams in the Mississippi Embayment in northern Arkansas and in Missouri, by P. R. Speer and others. 1966.
492. Thermal springs of the United States and other countries of the world--A summary, by G. A. Waring. 1965.

813-B. Summary appraisals of the Nation's groundwater resources--Upper Mississippi Region, by R. M. Bloyd, Jr. 1975.

813-H. Summary appraisals of the Nation's groundwater resources--Arkansas-White-Red Region, by M. S. Bedinger and R. T. Sniegocki. 1976.

937. The 1973 Mississippi River basin flood: Compilation and analyses of meteorologic, streamflow, and sediment data, by E. H. Chin and others. 1975.

WATER-SUPPLY PAPERS (WSP)

1139. Kansas-Missouri floods of July 1951. 1952.

1300. The industrial utility of public water supplies in the United States, 1952-Part 2, States west of the Mississippi River, by E. W. Lohr and S. K. Love. 1954.

1473. Study and interpretation of the chemical characteristics of natural water, 2d edition, by J. D. Hem. 1970.

1669-S. Yearly variations in runoff for the conterminous United States, 1931-60, by M.W. Busby. 1963.

1797. Has the United States enough water?, by A. M. Piper. 1965.

1800. The role of ground water in the national water situation, by C. L. McGuiness. 1963.

1812. Public water supplies of the 100 largest cities in the United States, 1962, by C. N. Durfor and Edith Becker. 1964.

216. Water resources of the St. Louis area, Missouri and Illinois, by J. K. Searcy, R. C. Baker, and W. H. Durum. 1952.

273. Water resources of the Kansas City area, Missouri and Kansas, by V. C. Fishel, J. K. Searcy, and F. H. Rainwater. 1953.

370. Floods in Missouri--Magnitude and frequency, by J. K. Searcy. 1955.

476. Principal lakes of the United States, by C. D. Bue. 1963.

536. Are we running out of water?, by R. L. Nace. 1967.
1838. Reservoirs in the United States, by R. O. R. Martin and R. L. Hanson. 1966.

1871. Water data for metropol itan areas--A summary of data from 222 areas in the United States, compile $\mathrm{i}$ by $W$. J. Schneider. 1968.

1899-I. Streamflow from the United States into the Atlantic Ocean during 1931-60, by C. D. Bue. 1970.

1990. Annotated bibliography on artificial recharge of ground water, 1955-67, by D. C. Signor, D. J. Growitz, and William Kam. 1970.

2020. Subsurface waste disposal by means of wells--A selective annotated bibliography, by $D$. R. Rima, E. B. Chase, and B. M. Myers. 1971.

2048. Water quality in the Ozark National Scenic Riverways, by J. H. Barks. 1978.

\section{CIRCULARS}

554. Hydrology for urban land planning--A guidebook on the hydrologic effects of urban land use, by L. B. Leopold. 1968.

566. Estimated use of water in the United States, 1965, by C. R. Murray. 1968.

601-A. Water for the cities--The outlook, by W. J. Schneider and A. M. Sp ieker. 1969.

601-D. Water as an urban resource and nuisance, by H. E. Thomas and W. J. Schneider. 1970.

601-E. Sediment problems in urban areas, by H. P. Guy. 1970. 
601-F. Hydrologic implications of solid-waste disposal, by W. J. Schneider. 1970.

601.G. Real-estate lakes, by D. A. Rickert. and A. M. Speiker. 1972.

$601-H$. Role of water in urban planning and management, by $W$. J. Schneider, D. A. Rickert, and A. M. Spieker. 1973.

$601-I$. Water facts and figures for planners and managers, by J. H. Feth. 1973.

691-J. Extent and development of urban flood plains, by W. J. Schneider and J. E. Goddard. 1974.

601-K. An introduction to the processes, problems, and management of urban lakes, by $L$. J. Britton, R. C. Averett, and R. F. Ferreira. 1975.

631. Disposal of liquid wastes by injection underground--Neither myth nor millenium, by A. M. Piper. 1969.

643. Reconnaissance of selected minor elements in surface waters of the United States, October 1970 , by W. H. Durum, J. D. Hem, and S. G. Heidel. 1971.

\section{HYDROLOGIC INVESTIGATIONS ATLASES (HA)}

61. Stream composition of the conterminous United States, by F. H. Rainwater. 1962.

315. Reconnaissance of the ground-water resources of the Missouri River alluvium between St. Charles and Jefferson City, Missouri, by L. F. Emmett and H. G. Jeffery. 1968 .

194. Generalized map showing annual runoff and productive aquifers in the conterminous United States, compiled by C. L. McGuinness. 1964.

199. Preliminary map of the conterminous United States showing depth to and quality of shallowest ground water containing more than 1,000 parts per million dissolved solids, by J. H. Feth and others. 1965.

200. Chemical quality of public water supplies of the United States and Puerto Rico, 1962, by C. N. Durfor and Edith Becker. 1964.

212. Annual runoff in the conterminous United States, by M. W. Busby. 1966.

217. General availability of ground water and depth to water level in the Missouri River basin, by G. A. LaRocque, Jr. 1966.

235. Temperature of surface waters in the conterminuous United States, by J. F. B?akey. 1966.

282. River discharge to the sea from the shores of the conterminous United States--A contribution to the International Hydrological Decade, compiled by Alfonso Wilson and others. 1967. pact, by L. B. Leopold, F. E. Clarke, B. B. Hanshaw, and J. R. Balsley. 1971.

670. Fluvial-sediment discharge to the oceans from the conterminous United States, by W. F. Curtis, J. K. Culbertson, and E. B. Chase. 1973.

676. Estimated use of water in the United States in 1970, by C. R. Murray and E. B. Reeves. 1972.

685. Dissolved-solids discharge to the oceans from the conterminous United States, by D. K. Leifeste. 1974.

703. Water demands for expanding energy development,

708. Model synthesis in frequency analys is of Missouri floods, by L. D. Hauth. 1974.

719. The National Stream Quality Accounting Network (NASQAN)--Some questions and answers, by J. F. Ficke and R. O. Hawkinson. 1975.
654. A procedure for evaluating environmental imby G. H. Davis and L. A. Wood. 1974.
332. Travel of solutes in the Lower Missouri River, by J. E. Bowie and L. R. Petri. 1969.

336. Reconnaissance of the ground-water resources of the Missouri River alluvium between Kansas City, Missouri and the Iowa border, by L. F. Emmett and H. G. Jeffery. 1969.

340. Reconnaissance of the ground-water resources of the Missouri River alluvium between Jefferson City and Miami, Missouri, by L. F. Emmett and H. G. Jeffery. 1969.

344. Reconnaissance of the ground-water resources of the Missouri River alluvium between Miami and Kansas City, Missouri, by L. F. Emmett and H. G. Jeffery. 1970.

372. Water resources of northeastern Missouri, by E. E. Gann and others. 1971.

444. Water resources of northwestern Missouri, by $E$. E. Gann and others. 1973.

491. Water resources of west-central Missouri, by E. E. Gann and others. 1974.

550. Water resources of south-central Missouri, by E. E. Gann and others. 1976. 
U.S. Geological Survey, 1976, hydrologic unit map of Missouri-1974.

\section{OPEN-FILE REPORTS OF THE U.S. GEOLOGICAL SURVEY}

Open-file reports are available for inspection in the Rolla, Mo., and Reston, Va., office of the U.S. Geological Survey; copies ordinarily are not reproduced for distribution. Further information may be obtained from the District Chief, water Resources Division, Rolla.

\section{Unnumbered Open-File Reports}

Bader, J. S., and others (compilers), 1973, Selected references--Ground-water contamination in the United States of America and Puerto Rico.

Bolon, H. C., 1953, Low-water study of the Gasconade River basin.

1955, Low-water study of the Spring River basin.

Bowie, J. E., 1971, Temperature of Missouri streams.

Gann, E. E., 1971, Generalized flood-frequency estimates for urban areas in Missouri.

Hauth, L. D., and Spencer, D. W., 1969, Floods in Gravois Creek basin in St. Louis County, Missouri.

1971, Floods in Coldwater Creek, Watkins Creek, and River des Peres basins, St. Louis County, Missouri.

Hauth, L. D., 1973, Selected storm events in 5-minute increments, from Missouri rainfall stations at Kansas City, St. Louis, Springfield, and Columbia.

1973, Rainfall-runoff data for smal1 drainage areas of Missouri.

1974, Technique for estimating the magnitude and frequency of Missouri floods.

\section{WATER-Resources InVestigations (WRI) REPORTS of the U.S, GEOLOgical SuRVEy}

Reports in this series are available for inspection at the Rolla, Mo., and Reston, Va., offices of the U.S. Geological Survey. Selected reports may be purchased either as microfilm or hard copy from the National Technical Information Service (NTIS), U.S. Department of Commerce, Springfield, Va.22161. Further information about these reports may be obtained from the District Chief, Water Resources Division, Rolla.

59-73. Estimating low-flow frequency for perennial Missouri Ozarks streams, by John Skelton. 1974.

78-63. Floods in Kansas City, Missouri and Kansas, September 12-13, 1977, by L. D. Hauth and W. J. Carswell, Jr. 1978.

77-16. Application of thermal imagery and aerial photography to hydrologic studies of karst terrane in Missouri, by $E$. J. Harvey, J. H. Williams, and T. R. Dinkel. 1977.

77-75. Effects of abandoned lead and zinc mines and tailings piles on water quality in the Jopl in area, Missouri, by J. H. Barks. 1977.
Jordan, P. R., 1968, Summary and analys is of sediment records in relation to St. Louis Harbor sedimentation problem.

Roemer, E. A., 1953, Low-water study of the Meramec River basin.

Skelton, John, and Homyk, Anthony, 1970, A proposed streamflow data program for Missouri.

Spencer, D. W., and Hauth, L. D., 1968, Floods in Mal ine Creek basin, St. Louis County, Missouri.

Spencer, D. W., 1971, Computed flood profile, River des Peres, Groby Street to $82 \mathrm{~d}$ Boulevard, University City, St. Lou is County, Missouri.

U.S. Geological Survey, 1961-64, Surface-water records of Missouri [published annually] 1964, Water-quality records in Missouri.

965-78, Water resources data for Missouri--Part 1, Surface-water records; Part 2, Water-quality records [published annually]
78-139. Technique for estimating the magnitude and frequency of floods in St. Louis County, Missouri, by D. W. Spencer and T. W. Alexander. 1978.

79-22. Water quality in the proposed Prosperity Reservoir area, Center Creek basin, Missouri, by J. H. Barks and W. R. Berkas. 1979. 


\section{Publications of the Missouri Division of Geology and Land Survey}

Many of these reports were prepared in cooperation with the U.S. Geological Survey. The reports may be obtained from the Director and State Geologist, who can furnish a more complete list of reports of the Division of Geology and Land Survey. They may be consulted in the following officies: Director and State Geologist, Division of Geology and Land Survey, Buehler Park, Rolla, Mo.; District Chief, Water Resources Division, U.S. Geological Survey, 1400 Independence Road, Rolla, Mo.; and Chief Hydrologist, U.S. Geological Survey, 420 National Center, Reston, Va. 22092.

Aley, T. J., and others, 1972, Ground-water contamination and sinkhole collapse in soluble rock terrain: Eng. Geology Ser. No. 5.

Barks, J. H., 1976, Water-quality characteristics of six small lakes in Missouri: Water Resources Rept. 33.

Beckman, H. C., 1927, Water resources of Missouri, 18571926: Missouri Bur. Geology and Mines, v. 20 2d ser.

1940, Water resources of Missouri, 1927-39: Missouri Bur, Geology and Mines, v. 26, 2d ser.

Beckman, H. C., and Hinchey, N. S., 1944, The large springs of Missouri: Missouri Bur. Geology and Mines, v. 29, 2d ser.

Bolon, H. C., 1952, Surface waters of Missouri: Missouri Bur. Geology and Mines, v. 34, 2d ser.

Bowie, J. E., and Gann, E. E., 1967, Floods of July $18-23,1965$, in northwestern Missouri: Water Resources Rept. 21.

Dean, T. J., and others, 1976, Guide for the geologic and hydrologic evaluation of small lake sites in Missouri: Water Resources Rept. 31.

Emmett, L. F., and others, 1978, Water resources of the Springfield, Missouri area: Water Resources Rept. 34.

Feder, G. L., and others, 1969, Water resources of the Jopl in area, Missouri: Water Resources Rept. 24.

Fuller, D. L., 1962, Ground-water-quality map of deep aquifers in Missouri in Ground-water maps of Missouri (published 1963).

(compiler), 1966, Lakes and reservoirs map of Missouri.

Groshskopf, J. G., 1955, Subsurface geology of the Mississippi Embayment of southeast Missouri: Missouri Bur. Geology and Mines, v. 37, 2d ser.

\section{GROUND-WATER REPORTS}

1-15. Water possibilities from the glacial drift of*** County. 1956-57: 1, Grundy County; 2, Mercer County; 3, Harrison County; 4, Putnam County; 5 , Worth County; 6 , Livingston County; 7 , Gentry County; 8 , DeDalb County; 9, Daviess County; 10, Sullivan County; 11, Linn County; 12, Chariton County; 13, Carroll County; 14, Buchanan County; 15, Andrew County.

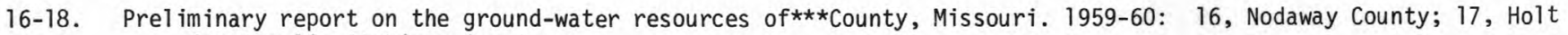

County; 18, Atchison County.
Heim, G. E., and Howe, W. B., 1962, Map of bedrock topography of northwestern Missouri in Ground-water maps of Missouri (published 1963).

Knight, R. D., 1962, Ground-water areas in Missouri in Ground-water maps of Missouri (published 1963).

McCracken, M. H., 1971, Structural features of Missouri: Rept. of Investigations No. 49.

Miller, D. E., and others, 1974, Water resources of the St. Louis area, Missouri: Water Resources Rept. 30.

Miller, J. C., 1971, Ground-water resources of Saline County, Missouri: Water Resources Rept. 26.

Petersen, M. S., 1965, Floods of June 17th and 18th, 1964, in Jefferson, St. Genevieve, and St. Francois Counties, Missouri: Water Resources Rept. 19.

Robertson, C. E., 1962, Water well yield map of Missouri in Ground-water maps of Missouri (published 1963).

Sandhaus, E. H., and Skelton, John, 1968, Magnitude and frequency of Missouri floods: Water Resources Rept. 23.

Skelton, John, 1966, Low-flow characteristics of Missouri streams: Water Resources Rept. 20.

1968, Storage requirements to augment low flows of Missouri streams: Water Resources Rept. 22.

1970, Base-flow recession characteristics and seasonal low-flow frequency characteristics for Missouri streams: Water Resources Rept. 25.

1971, Carryover storage requirements for reservoir design in Missouri: Water Resources Rept. 27.

1976, Missouri stream and springflow characteristics-Low-flow frequency and flow duration: Water Resources Rept. 32.

Vineyard, J. D., and Feder, G. L., 1974, Springs of Missouri: Water Resources Rept. 29. 


\section{ADDRESS INQUIRES ABOUT THE AVAILABILITY OF THESE REPORTS TO THE PUBLISHER,}

Clifford, H. F., 1966, Some 1 imnological characteristics of six 0zark streams: Missouri Dept. of Conservation.

Ryck, F. M., 1974, Missouri stream pollution survey: Missouri Dept. of Conservation.

1974, Water quality survey of the Southeast Ozark mining area: Missouri Dept. of Conservation.

Taylor, C. T., 1964, Chemical quality of Missouri surface water: Missouri Dept. Publ ic Health and Welfare, Water Pollution Board [currently the Dept. of Natural Resources, Div. of

Environmental Quality (Clean Water Commission)].
Taylor, C. T., 1964, Water quality of Big, Bourbeuse, and Meramec River basins: Missouri Dept. Public Health and Welfare, Water Pollution Board [currently the Dept. of Natural Resources, Div. of Environmental Quality (Clean Water Commission)].

U.S. Geological Survey, and Missouri Geological Survey and Water Resources, 1967, Mineral and water resources of Missouri: 90th Cong., 1st sess. Senate Doc. 19. [Also available from Missouri State Geologist]. 

